\title{
References in Chronological Order
}

1. R. HUISGEN, H. WIELAND - Über Strychnos-Alkaloide, XXIX. Die Konstitution des Desoxyvomicins. Liebigs Ann. Chem. 555, 9-25 (1943).

2. H. WIELAND, R. HUISGEN - Über Strychnos-Alkaloide, XXX. Weitere Studien am Vomicin. Liebigs Ann. Chem. 556, 157-170 (1944).

3. R. HUIS GEN - Die Konstitution der Strychnos-Alkaloide. Die Pharmazeut. Industrie $11,383-388$ (1944).

4. R. HUISGEN - Über Strychnos-Alkaloide, XXXI. Weitere Synthesen in der Reihe des 5.6(N)-Ryrrochinolins. Liebigs Ann. Chem. 559, 174-190 (1948).

5. H. WIELAND, R. HUISGEN, R. BUBENIK - Über Strychnos-Alkaloide, XXXIt. Strychnin und Vomicin. Liebigs Ann. Chem. 559, 191-199 (1948).

6. R. HUISGEN - Beiträge und Deutungsversuche zur Reaktionsweise aromatischer Bicyclen. Liebigs Ann.Chem. 559, 101-152 (1948).

7. R. HUISGEN, H. WIELAND, H. EDER - Über Strychnos-Alkaloide, XXXIII. Abbouversuc he an quartären Salzen des Vomicins und Desoxyvomicins. Liebigs Ann. Chem. $561,193-215$ (1949).

8. R. HUISGEN, G. HORELD - Nitroso-acyl-amine und Diazoester, I. Die Phenylierung aromatischer Verbindungen mit Nitroso-acyl-aniliden. Liebigs Ann.Chem. 562, 137-162 (1949).

9. R. HUISGEN - Orientierungsphänomene bei der Substitution aromat ischer Bicyclen, II. Die Chinolinsynthese nach COMBES. Liebigs Ann.Chem. 564, 16-32 (1949).

10. R. HUISGEN - Beiträge zUm Radikalzerfall aromatischer Diazoverbindungen. Angew. Chem. 61,389 (1949).

11. R. HUISGEN - Die Strychnos-Alkaloide. Ein Überblick Uber ein halbes Jahrhundert Alkaloidforschung. Angew. Chem. 62, 527-534 (1950). 
12. R. HUISGEN - Zur Stereochemie der aromatischen Diazoverbindungen. Angew. Chem. $62,369(1950)$.

13. R. HUISGEN, G. SORGE - Orientierungsphänomene bei der Substitution aromatischer Bicyclen, 11l. Radikalsubstitutionen in der Naphthalinreihe. Liebigs Ann. Chem. 566, 162-184 (1951).

14. R. HUISGEN, H. EDER, L. BLAZEJEWICZ, E. MERGENTHALER - Über StrychnosAlkaloide, XXXIV. Die Isomeren des Desoxyvomicins und ihre katalytische Hydrierung. Liebigs Ann. Chem. 573, 121-141 (1951).

15. R. HUISGEN - Nitroso-acyl-amine und Diazoester, II. Acylwanderung und Acylablösung be i Nitroso-acyl-aniliden. Liebigs Ann. Chem. 573, 163-181 (1951).

16. R. HUISGEN, H. NAKATEN - Nitroso-acyl-amine und Diazoester, III. Die Reaktion von Nitroso-acyl-aniliden mit Alkohol. Liebigs Ann. Chem. 573, 181-195 (1951).

17. R. HUISGEN, L. KRAUSE - Nitroso-acyl-amine und Diazoester, IV. Die Konfiguration der Diazoester und der Mechanismus ihrer Bildung durch Acylwanderung. Liebigs Ann. Chem. 574, 157-171 (1951).

18. R. HUISGEN - Nitroso-acyl-amine und Diazoester, V. Aromatische Nitroso-lactome und cyclische Diazoester. Liebigs Ann. Chem. 574, 171-184 (1951).

19. R. HUISGEN - Nitroso-acyl-amine und Diazoester, VI. Die Reaktion von Nitrosoacyl-aniliden mit Carbonsuluren. Liebigs Ann. Chem. 574, 184-201 (1951).

20. R. HUISGEN, J. REINERTSHOFER - Ein never Aspekt der Chemie mittlerer Ringe. Z. Naturforsch., Teil B, 6,395 (1951).

21. R. HUISGEN, J. REINERTSHOFER - Nitroso-ocyl-amine und Diazoester, VII. Nitroso-caprolactom und seine Reaktionen. Liebigs Ann. Chem. 575, 174-197 (1952).

22. R. HUISGEN, 1. REINERTSHOFER - Nitroso-acyl-amine und Diazoester, VIII. Aliphat ische Nitroso-lactame und cyclische Diazoester. Liebigs Ann. Chem. 575, 197-216 (1952). 
23. R. HUISGEN, W. RAPP - Mittlere Ringe, 1. 1,2-Benzo-cycloocten-(1)-on-(3). Chem. Ber. 85, 826-835 (1952).

24. R. HUISGEN - Liebigs unvergangliches chemisches Werk. Angew. Chem. 65,361 365 (1953).

25. R. HUISGEN - Neues Uber aliphatische Diazo- und Azoverbindungen. Angew. Chem. 65, 40 (1953).

26. R. HUISGEN - Darstellung und Eigenschaften mittlerer Ringe. Angew. Chem. 65, 540 (1953).

27. R. HUISGEN, H.J. KOCH - Die Reaktion von p-Nitro-benzoldiazonium-chlorid mit Diazomethan; eine neue Sextettumlagerung. Naturwissenschaften 41, 16 (1954).

28. R. HUISGEN, W. RAPP, I. UGI, H. WALZ, E. MERGENTHALER - Mittlere Ringe, II. Sterische Hinderung der Mesomerie in 1,2-Benzo-cyclen-3-onen und verwandten Verbindungen. Liebigs Ann. Chem. 586, 1-29 (1954).

29. R. HUISGEN, 1. UGI, H. BRADE, E. RAUENBUSCH. Mittlere Ringe, III. Eigenschaften und Reaktionen der 1,2-Benz-lactame. Liebigs Ann. Chem. 586, 30-51 (1954).

30. R. HUISGEN, W. RAPP, I. UGI, H. WALZ, I. GLOGGER - Mittlere Ringe, IV. Darstellung und Eigenschaften der 1,2,3,4-Benzo-cyclo-1,3-dienone-(5). Liebigs Ann. Chem. 586, 52 (1954).

31. R. HUISGEN, H. NAKATEN - Zum Radikalzerfall des Phenyl-azo-triphenylmethans. Liebigs Ann. Chem. 586, 70-83 (1954).

32. R. HUISGEN, H. NAKATEN - Nitroso-acyl-amine und Diazoester, IX. Die JACOBSON sche Indazolsynthese und die Konfiguration der Diazoester. Liebigs Ann. Chem. 586, 84-109 (1954).

33. K. CLUSIUS, H. HÜRZELER, R. HUISGEN, H.J. KOCH - Zur Reaktion des p-Nitro-benzoldiazonium-chlorids mit Diazomethan; Prufung des Reaktionsablaufs mit schwerem Stickstoff. Naturwissenschaften 41, 213 (1954). 
34. R. HUISGEN - Neve Reaktionen aliphatischer Diazo- und Azoverbindungen. Österr. Chem. -Zrg. 55, 237-245 (1954).

35. R. HUISGEN, H. RIST - Über Umlagerungen bei nucleophilen Substitutionen in der aromatischen Reihe und ihre Deutung. Naturwissenschaften 41, 358-359 (1954).

36. R. HUISGEN, F. JAKOB, W. SIEGEL, A. CADUS - Additionsreaktionen der NNDoppelbindung, 1. Die Anlagerung der aromatischen Seitenkette an Azodicarbonester. Liebigs Ann. Chem. 590, 1-36 (1954).

37. R. HUIS GEN, F. JAKOB - Additionsreaktionen der NN-Doppelbindung, II. Der Chemismus einiger weiterer Reaktionen des Azodicarbonester. Liebigs Ann. Chem. 590, 37-54 (1954).

38. R. HUISGEN - Modeme Chemie der Kohlenwasserstoffe. Prakt. Schulphysik 30 , 343-356 (1954).

39. R. HUISGEN, H.J. KOCH - Die Kupplung aromatischer mit aliphatischen Diazoverbindungen. Liebigs Ann. Chem. 591, 200-231 (1955).

40. R. HUISGEN, H. RIST - Nucleophile aromatische Substitutionen, I. Die Reaktion aromatischer Fluorverbindungen mit Phenyllithium; ein Beitrag zum Chemismus der Umlage rungen bei nucleophilen aromatischen Substitutionen. Liebigs Ann. Chem. 594, 137-158 (1955).

41. R. HUISGEN, H. RIST - Nucleophile aromatische Substitutionen, II. Die Reaktion von Nitrobenzol mit N-Lithium-piperidin. Liebigs Ann. Chem. 594, 159-165 (1955).

42. R. HUIS GEN - Altes und Neves Uber aliphatische Diazoverbindungen. Angew. Chem. 67, 439-463 (1955).

43. R. HUISGEN, D. VOSSIUS, M. APPL - Abkömmlinge des Tropazols. Angew. Chem. 67, 756-757 (1955).

44. R. HUISGEN, W. RAPP, U. RIETZ, E. RAUENBUSCH, V. TRESCHER, I. UGI, V. VOSSIUS, H. WALZ - Die Synthese mittlerer und großer Ringe mit Hilfe der intramolekularen Acylierung nach Friedel-Crafts. Angew. Chem. 67, 757 (1955). 
45. R. HUISGEN - Ausfuhrung kinetischer Versuche. Hondbuchartikel in HOUBENWEYL, Methoden der Organischen Chemie, 4. Aufl., Bd. 111/1, S. 101-162 (1955).

46. R. HUISGEN, H. WALZ - Mittlere Ringe, V. Die elektrischen Momente der aliphat ischen Lactame und die Konfiguration der Stureamidgruppe. Chem. Ber. 89, 2616-2629 (1956).

47. R. HUISGEN, H. REIMLINGER - Nitroso-acyl-amine und Diazoester, X. Die Isomerisierung der Nitroso-acyl-alkylamine zu Diazoestem und ihre Kinetik. Liebigs Ann. Chem. 599, 161-182 (1956).

48. R. HUISGEN, H. REIMLINGER - Nitroso-acyl-amine und Diazoester, XI. Der Zerfall der Alkyldiazoester in organischen Lösungsmitteln. Liebigs Ann. Chem. 599, 183-202 (1956).

49. R. HUISGEN, C. RÜCHARDT - Nitroso-acyl-amine und Diazoester, XIl. Zur Frage der Carbonium-Umlagerungen beim Zerfall der Alkyldiazoester. Liebigs Ann. Chem. 601, 1-21 (1956).

50. R. HUISGEN, C. RÜCHARDT - Nitroso-acyl-amine und Diazoester, XIII. Zum sterischen Ablauf des Alkyldiazoester-Zerfolls. Liebigs Ann.Chem. 601, 21-39 (1956).

51. R. HUISGEN, I. UGI - Zur Lösung eines klassischen Problems der organischen Stickstoff-Chemie. Angew. Chem. 68, 705-706 (1956).

52. R. HUISGEN - Neue Beiträge zur organischen Stickstoff-Chemie. Chimia 10, 266-267 (1956).

53. I. UGI, R. HUISGEN, K. CLUSIUS, M. VECCHI - Zur Reaktion des Benzoldiazonium-lons mit Azid; Nachweis des Phenyl-pentazols ols Zwischenstufe. Angew. Chem. 68, 753-754 (1956).

54. R. HUISGEN, J. SAUER, A. HAUSER - Katalytische Phenylierung der Chlor-Aromaten mit Phenyllithium. Angew. Chem. 69, 267-268 (1957).

55. R. HUISGEN, H. KÖNIG - Ringschlußreaktionen Uber Arine. Angew. Chem. 69, 268 (1957). 
56. R. HUISGEN, L. ZIRNGIBL - Addition von Basen an 1.2-Naphthin. Angew. Chem. $69,389-390$ (1957).

57. R. HUISGEN, J. SAUER - Reaktion der Halogen-naphthaline mit Lithium-piperidid. Angew. Chem. 69, 390 (1957).

58. R. HUISGEN, I. UGI, M.T. ASSEMI, J. WITTE - Mittlere Ringe, VI. Die Kinetik der Beckmann-Umlagerung der Cyclanon-oxime. Liebigs Ann. Chem. 602, 127135 (1957).

59. R. HUISGEN, J. WITTE, H. WALZ, W. JIRA - Zum Chemismus der Arylwanderung bei der Beckmann-Umlagerung, 1. Liebigs Ann. Chem. 604, 191-202 (1957).

60. R. HUISGEN, J. WITTE, I. UGI - Zum Chemismus der Arylwanderung bei der Beckmann-Umlagerung, II. Chem. Ber. 90, 1844-1849 (1957).

61. R. HUISGEN, J. WITTE, W. JIRA - Weitere kinetische Untersuchungen zur Beckmann-Umlagerung der Aryl-alkyl-ketone. Chem. Ber. 90, 1850-1856 (1957).

62. R. HUISGEN, H. BRADE, H. WALZ, I. GLOGGER - Mittlere Ringe, VII. Die Eigenschaften aliphatischer Lactame und die cis,trans-Isomerie der Sdureamidgruppe. Chem. Ber. 90, 1437-1447 (1957).

63. R. HUISGEN - Mittlere Ringe, VIII. Neve Beiträge zur Chemie mittlerer Ringe. Angew. Chem. 69, 341-359 (1957).

64. R. HUISGEN, G. SEIDL - Neue Umlagerung bei Solvolysen in der 1,2-Benzocyclenyl-(3)-methyl-Reihe. Angew. Chem. 69, 390-391 (1957).

65. R. HUISGEN, H. BRADE - Die Basizitartskonstanten offenkettiger Cabonsüureamide. Chem. Ber. 90, 1432-1436 (1957).

66. R. HUISGEN, 1. UGI, E. RAUENBUSCH, V. VOSSIUS, H. OERTEL - Mittlere Ringe, IX. Weiteres zur sterischen Mesomeriehinderung in 1,2-Benzo-cyclen-(1)onen-(3). Chem. Ber. 90, 1946-1958 (1957).

67. R. HUISGEN, E. RAUENBUSCH, G. SEIDL - Mittlere Ringe, X. Reaktionen in der Reihe des 1,2-Benzo-cyclooctens-(1). Chem. Ber. 90, 1958-1965 (1957). 
68. R. HUISGEN, 1. UGI - Mittlere Ringe, XI. Polycyclische Systeme mit Heterootomen. Liebigs Ann. Chem. 610, 57-66 (1957).

69. R. HUISGEN, V. VOSSIUS - Mittlere Ringe, XII. Die intramolekulare Acylierung der $\omega$-(p-Tolyl)-n-fettsduren. Monatsh. Chem. 88, 517-540 (1957).

70. R. HUISGEN, U. RIETZ - Mittlere Ringe, XIV. Darstellung und Cyclisierung der $\omega$-(Naphthyl-(2))-fettsduren. Chem. Ber. 90, 2768-2784 (1957).

71. R. HUISGEN - Heinrich Wieland - 80 Jahre; zum 4.6.1957. Naturwissenschaften 44, $317-318$ (1957).

72. R. HUISGEN, R. GRASHEY - Phenylierungen aromatischer Verbindungen mit Phenylazo-triphenylmethan und anderen Phenylgeneratoren. Liebigs Ann. Chem. 607, 46-59 (1957).

73. R. HUIS GEN, J. SAUER, A. HAUSER, H. KÖNIG, L. ZIRNGIBL - Zur Kenntnis nucleophiler Substitutionen über Arine. Angew. Chem. 69, 682 (1957).

74. R. HUISGEN, I. UGI - Pentazole, 1. Die Lösung eines klassischen Problems der organischen Stickstoff-Chemie. Chem. Ber. 90, 2914-2927 (1957).

75. R. HUISGEN, D. VOSSIUS, M. APPL - Die Thermolyse des Phenylazids in Primären Aminen; die Konstitution des Dibenzamils. Chem. Ber. 91, 1-12 (1958).

76. R. HUISGEN, M. APPL - Der Chemismus der Ringerweiterung beim Zerfall des Phenylazids in Anilin. Chem. Ber. $91,12-21$ (1958).

77. I. UGI, R. HUISGEN - Pentazole, II. Die Zerfallsgeschwindigkeit der Arylpentazole. Chem. Ber. 91, 531-537 (1958).

78. R. HUISGEN, U. RIETZ - Mittlere Ringe, XIII. Die intramolekulare Acylierung der $w$-(1-Naphthyl)-fettsduren. Tetrahedron 2, 271-288 (1958).

79. R. HUISGEN, J. SAUER, H.J. STURM - Acylierung 5-substituierter Tetrozole zU 1,3,4-Oxadiazolen. Angew. Chem. 70, 272-273 (1958). 
80. R. HUISGEN, H. OTT - Darstellung und Konfiguration von Heptanolid und Octanolid. Angew. Chem. 70, 312 (1958).

81. R. HUISGEN, J. WITTE - Eine neue Synthese von N-substituierten Thiocarbonsdureamiden. Chem. Ber. 91, 972-977 (1958).

82. J. WITTE, R. HUISGEN - Zur Überfuhrung N-substituierter Carbonamide in Thioamide. Chem. Ber. $91,1129-1131$ (1958).

83. R. HUISGEN, L. ZIRNGIBL - Nucleophile aromatische Substitutionen, III. Weiteres zur Reaktion der Fluor-naphthaline mit lithiumorganischen Verbindungen. Chem. Ber. $91,1438-1452$ (1958).

84. R. HUISGEN, J. SAUER - Nucleophile aromatische Substitutionen, IV. Umsetzungen der Halogen-benzole und -tolvole mit Lithium-piperidid in $\ddot{A}$ ther. Chem. Ber. 91, 1453-1460 (1958).

85. J. SAUER, R. HUISGEN, A. HAUSER - Nucleophile aromatische Substitutionen, V. Der Chemismus der Reaktion der Halogen-naphthaline mit Lithium-piperidid und Lithium-diathylamid. Chem. Ber. 91, 1461-1473 (1958).

86. R. HUISGEN, J. SAUER, M. SEIDEL - A New Synthesis of 1:2:4-Tetrazoles: The Reaction of 5-Substituted Tetrazoles with Imino-chlorides. Chem. Ind. (London), 1958, 1114-1115.

87. R. HUISGEN - The Wieland Memorial Lecture. Proc. Chem. Soc. (London), 210219 (1958).

88. R. HUISGEN, J. SAUER, A. HAUSER - Nucleophile aromatische Substitutionen, VI. Katalytische Arylierung der Chloraromaten. Chem. Ber. 91, 2366-2374 (1958).

89. R. HUISGEN, L. ZIRNGIBL - Nucleophile aromatische Substitutionen, VII. Sterische und elektronische Faktoren bei der Basen-Addition an 1,2-Naphthin. Chem. Ber. 91, 2375-2382 (1958).

90. R. HUISGEN, J. SAUER - Nucleophile oromatische Substitutionen, VIII. Kinetik der Freisetzung des Benz-ins aus Halogenbenzolen. Chem. Ber. 92, 192-202 (1959). 
91. R. HUISGEN, H. KÖNIG - Nucleophile aromatische Substitutionen, IX. Ringschlußreaktionen zu N-Methyl-indolin und $\mathrm{N}$-Methyl-tetrahydrochinolin und ihr Chemismus. Chem. Ber. 92, 203-213 (1959).

92. R. HUISGEN, H. KÖNIG, N. BLEEKER - Nucleophile aromatische Substitutionen, X. Kinetik der Ringschlußreaktionen zu N-Methyl-indolin und N-Methyl-tetrahydrochinolin. Chem. Ber. 92, 424-429 (1959).

93. H. KÖNIG, R. HUISGEN - Nucleophile aromatische Substitutionen, XI. Weitere Ringschlußreaktionen über Arine. Chem. Ber. 92, 429-441 (1959).

94. R. HUISGEN, J. SAUER, W. MACK, I. ZIEGLER - Nucleophile aromatische Substitutionen, XII. Konkurrierende Substitutionsmechanismen bei den Fluornaphthalinen. Chem. Ber. 92, 441-449 (1959).

95. R. HUISGEN - Heinrich Wieland zum Gedøchtnis; Heinrich Wieland als Mensch und Lehrer. Angew. Chem. 71, 5-6 (1959).

96. R. HUISGEN - Recent Developments in the Elimination Mechanism of Nucleophilic Aromatic Substitution. Kekulé-Symposium, London 1958. Theoretical Organic Chemistry, Butterworth Scientific Publications, London, 158-179 (1958).

97. R. HUISGEN, H. FLEISCHMANN - Reaktionen aromatischer mit aliphatischen Diazoverbindungen, II. Die Azokupplung der Chinondiazide mit aliphatischen Diazoverbindungen. Liebigs Ann. Chem. 623, 47-68 (1959).

98. R. HUISGEN, H. OTT - Die Konfiguration der Carbonestergruppe und die Sondereigenschaften der Lactone. Tetrahedron 6, 253-267 (1959).

99. R. HUISGEN, F. JAKOB, R. GRASHEY - Konkurrenzkonstante und partielle Geschwindigkeitsfaktoren bei der radikal ischen Phenylierung des Naphthalins. Chem. Ber. 92, 2206-2210 (1959).

100. R. GRASHEY, R. HUISGEN - Die Reaktion des Phenylazotriphenylmethans mit Ryridin; ein Beitrog zur Frage der Zwischenstufe bei der radikalischen Phenylierung. Chem. Ber. 92, 2641-2645 (1959). 
101. R. HUISGEN, M. SEIDEL, J. SAUER, J.W. McFARLAND, G. WALlBILLICH The Formation of Nitrile Imines in the Thermal Breakdown of 2,5-Disubstituted Tetrazoles. J.Org. Chem. 24, 892-893 (1959).

102. M. APPL, R. HUISGEN - Weiteres zur Thermolyse der Arylazide in Aminen. Chem. Ber. 92, 2961-2967 (1959).

103. R. HUISGEN, F. BAYERLEIN, W. HEYDKAMP - Die Acylierung der N, N-Dimethylarylamin-oxyde; der Mechan ismus der Polonovski-Reaktion. Chem. Ber. 92, 3223-3241 (1959).

104. F. BAYERLEIN, R. HUISGEN - Notiz Uber einige weitere Entmethylierungen der N, N-Dimethy larylamine Uber das N-Oxyd. Chem. Ber. 92, 3242-3243 (1959).

105. R. HUISGEN, E. LASCHTUVKA - Eine neve Synthese von Derivaten des Ryrrols. Chem. Ber. 93, 65-81 (1960).

106. E. LASCHTUVKA, R. HUISGEN - Recktionen in der Fluorazon-Reihe. Chem. Ber. $93,81-88(1960)$.

107. R. HUISGEN, W. MACK - Nucleophile aromatische Substitutionen, XIII. Der Nachweis lossicher Komplexe aus Lithiumpiperidid und Lithiumhalogeniden. Chem. Ber. 93, 332-340 (1960).

108. R. HUISGEN, W. HEYDKAMP, F. BAYERLEIN - Zur Reaktion der N, N-Dimethylarylamine mit Diacyl-peroxyden; der Mechanismus der Entmethylierung. Chem. Ber. 93, 363-374 (1960).

109. R. HUISGEN, E. LASCHTUVKA, F. BAYERLEIN - 2,3,6,7-Dibenzo-azepin. Chem. Ber. 93, 392-397 (1960).

110. R. HUISGEN, W. MACK, K. HERBIG, N. OTT, E. ANNESER - Nucleophile aromatische Substitutionen, XIV. Partielle Geschwindigkeitskonstanten der Arin-Bildung aus Bromaromaten mittels Lithiumpiperidids. Chem. Ber. 93, 412-424 (1960).

111. R. HUISGEN, H. POHL - Additionsreaktionen der NN-Doppelbindung, III. Die Reaktion des Azodicarbonstureesters mit Olefinen. Chem. Ber. 93, 527-540 (1960). 
112. R. HUISGEN, R. LUX - Additionsreaktionen der NN-Doppelbindung, IV. Zum Mechanismus der Thenylhydrazinsynthese nach E. FISCHER. Chem. Ber. 93, 540544 (1960).

113. W. MACK, R. HUISGEN - Nucleophile aromatische Substitutionen, XVI. Lagem sich Lithiumdialkylamide oder freie sek. Amine an Arine an ? Chem.Ber. 93, 608613 (1960).

114. R. HUISGEN, J. SAUER - Nucleophile aromatische Substitutionen, XVII. Nucleophile aromatische Substitutionen Uber Arine. Angew. Chem. 72, 91-108 (1960).

115. J. SAUER, R. HUISGEN - Nucleophile aromat ische Substitutionen mit additivem Chemismus. Angew. Chem. 72, 294-315 (1960).

116. R. HUISGEN, W. MACK, E. MÖBIUS - Nucleophile aromatische Substitutionen, $X V$. Der Nachweis der Zwischenstufe bei nucleophilen aromatischen Substitutionen mit Eliminierung; zur Struktur der Arine. Tetrahedron 9, 29-39 (1960).

117. R. HUISGEN, E. LASCHTUVKA, I. UGI, A. KAMMERMEIER - Syntheseversuche in der Oxepin- und Azepin-Reihe. Liebigs Ann. Chem. 630, 128-138 (1960).

118. R. HUISGEN, F. BAYERLEIN - Die Acylierung N,N-disubstituierter Hydroxylamine und die Umsetzung sekundärer Amine mit Diacylperoxyden. Liebigs Ann. Chem. $630,138-146(1960)$.

119. R. HUISGEN - Thermische Stabilitat und aromatischer Charakter; Ringöffnungen der Azole. Angew. Chem. 72 , 359-372 (1960).

120. R. HUISGEN, R. GRASHEY, P. LAUR, H. LEITERMANN - 1,3-Dipolare Additionen der Azomethin-imine. Angew. Chem. 72, 416-417 (1960).

121. R. HUISGEN, R. FLEISCHMANN, A. ECKELL - Azomethin-imine, eine neve Klasse zwitterionischer Verbindungen. Tetrahedron Lett. 12, 1-4 (1960).

122. R. HUIS GEN, A. ECKELL - 1,3-Dipolare Additionen der Azomethin-imine. Tetrahedron Lett. $12,5-8$ (1960). 
123. R. GRASHEY, R. HUISGEN, H. LEITERMANN - 1,3-Dipolare Additionen der Nitrone. Tetrahedron Lett. 12, 9-13 (1960).

124. R. HUISGEN, H. KÖNIG, A. R. LEPLEY - Nucleophile aromatische Substitutionen, XVIII. Neue Ringschlusse Uber Arine. Chem. Ber. 93, 1496-1506 (1960).

125. R. HUISGEN - Theoretische Chemie und Organische Synthese. Zehnjahresfeier des Fonds der Chemischen Industrie, Dusseldorf, 73-102 (1960); Nachdruck in Naturwis5. Rundschau 14, 63 (1961).

126. R. HUISGEN, J. SAUER, H.J. STURM, J.H. MARKGRAF - Ringöffnungen der Azole, Il. Die Bildung von 1,3,4-Oxdiazolen bei der Acylierung 5-substituierter Tetrazole. Chem. Ber. 93, 2106-2124 (1960).

127. R. HUISGEN, I. UGI - Mittlere Ringe, XVI. Eigenschaften und Konfiguration der 1,4-Benzlactame. Chem. Ber. 93, 2693-2704 (1960).

128. R. HUISGEN - Benzyne Chemistry, in "Organometallic Chemistry" (Editor H.H. Zeiss). American Chemical Society Monograph Series. Reinhold Publ. Corp., New York, Chopman u. Hall, Ltd. London, 36-87 (1960).

129. J. SAUER, R. HUISGEN, H.J. STURM - Ringöffnungen der Azole, III. Zur Acylierung von 5-Aryl-tetrazolen; ein Duplikationsverfahren zur Darstellung von Polyarylen. Tetrahedron 11, 241-251 (1960).

130. R. HUISGEN, J. SAUER, M. SEIDEL - Ringöffnungen der Azole, IV. Die Synthese von 1,2,4-Triazolen aus 5-substituierten Tetrazolen und Corbonsdure-imidchloriden. Chem. Ber. 93, 2885-2891 (1960).

131. R. HUISGEN - R. Willstatter. J.Chem. Educ. 38, 10-16 (1961).

132. R. HUISGEN - Die Bedeutung der Theoretischen Organischen Chemie. Chemie fur Labor und Betrieb 12, 1 (1961).

133. R. HUISGEN, H. STANGL, H. J. STURM, H. WAGENHOFER - Kinetik und Mechanismus der 1,3-Dipolaren Additionen der Diazoalkane. Angew. Chem. 73, 170 (1961). 
134. R. HUISGEN, E. RAUENBUSCH - Mittlere Ringe, XVII. Zur Darstellung von $\omega$-(2-Carboxy-phenyl)-fettsduren durch Kettenverlangerung arylaliphatischer Sauren. Liebigs Ann. Chem. 641, 51-62 (1961).

135. I. UGI, R. HUISGEN, D. PAWELLEK - Mittlere Ringe, XVIII. Die Synthese von $\omega$-(2-Carboxy-phenyl)-fettsäuren durch nitrosierende Spaltung von Derivaten des a-Tetralons. Liebigs Ann. Chem. 641, 63-70 (1961).

136. R. HUISGEN, D. PAWELLEK - ZUr Darstellung aliphatischer Dicarbonsduren durch nitrosierende Spaltung von Cyclanon-Derivaten. Liebigs Ann. Chem. 641, 71-75 (1961).

137. R. HUISGEN, H.J. STURM, M. SEIDEL - Ringöffnungen der Azole, V. Weitere Reaktionen der Tet razole mit elektrophilen Agentien. Chem. Ber. 94, 1555-1562 (1961).

138. R. HUISGEN, H. KÖNIG, G. BINSCH, H.J. STURM - 1,3-Dipolare Additionen der Ketocarbene. Angew. Chem. 73, 368-371 (1961).

139. R. HUISGEN, G. JUPPE - Benzo-norcaradien-cabonsdure und Benzo-cycloheptatrien-carbonsäure. Chem. Ber. 94, 2332-2349 (1961).

140. R. HUISGEN, G. JUPPE - Umsetzungen des Diazoessigesters mit 2,3-Dimethylund 2-Methyl-naphthalin. Liebigs Ann. Chem. 646, 1-9 (1961).

141. R. HUISGEN, J. SAUER, M. SEIDEL - Ringöffnungen der Azole, VI. Thermolyse 2,5-disubstituierter Tetrazole zu Nitriliminen. Chem. Ber. 94, 2503-2509 (1961).

142. R. HUISGEN, M. SEIDEL - Ringöffnungen der Azole, VII. Thermolyse des 1-Benzoyldiphenyl-1,2,3-triazols. Chem. Ber. 94, 2509-2511 (1961).

143. R. HUISGEN, W. MACK, E. ANNESER - 1,3-Dipolare Additionen der Nitriloxide an CS-Doppelbindungen. Angew. Chem. 73, 656-657 (1961).

144. R. HUISGEN - 1,3-Dipolar Cycloadditions (Centenary Lecture, 8.12.1960, London). Proc. Chem. Soc., London, 1961, 357-369. 
145. R. HUISGEN, W. MACK - 1,3-Dipolare Additionen der Nitriloxide an Corbonylverbindungen. Tetrahedron Lett. 583 (1961).

146. R. HUISGEN, W. MACK, E. ANNESER - Synthese von 1,2,4-Oxdiazolen aus Nitriloxiden und Nitrilen. Tetrahedron Lett. , 587 (1961).

147. R. HUISGEN, G. JUPPE - Reaktion von 2,6-Dimethyl-naphthalin mit Diazoessigester. Tetrahedron 15, 7-17 (1961).

148. R. HUIS GEN, V. WEBERNDÖRFER - 1,3-Dipolare Additionen der Thioketocarbene. Experientia 17, 566 (1961).

149. R. HUISGEN, R. KNORR - Benz-in als Dipolarophil. Naturwissenschaften 48,716 (1961).

150. R. HUISGEN, R. GRASHEY, H. GOTTHARDT, R. SCHMIDT - 1,3-Dipolare Additionen der Sydnone an Alkine. Angew. Chem. 74, 29-30 (1962); Angew. Chem. int. Ed. Engl. 1, 48 (1962).

151. R. HUISGEN, H. GOTTHARDT, R. GRASHEY - Reaktionen der Sydnone mit Alkenen. Angew. Chem. 74, 30 (1962); Angew. Chem. Int. Ed. Engl. 1, 49 (1962).

152. R. HUIS GEN, H. STANGL, H.J. STURM, H. WAGENHOFER - 1,3-Dipolare Additionen mit Nitril-yliden. Angew. Chem. 74, 31 (1962); Angew. Chem., Int. Ed. Engl. 1, 50 (1962).

153. R. HUISGEN, M. SEIDEL, G. WALLBILLICH, H. KNUPFER - 1,3-Dipolare Additionen, 1. Diphenyl-nitrilimin und se ine 1,3-Dipoloren Additionen an Alkene und Alkine. Tetrahedron 17, 3-29 (1962).

154. R. HUISGEN, H.J. STURM, H. WAGENHOFER - Ein neues Kriterium fur Mehrzentren-Additionen zu funfgliedrigen Ringen. Z. Naturforsch., Teil B, 17, 202-203 (1962).

155. R. HUISGEN, R. GRASHEY, M. SEIDEL, G. WALLBILLICH, R. SCHMIDT - 1,3-Dipolare Additionen, 2. Synthese von 1,2,4-Triazolen aus Nitriliminen und Nitrilen. Liebigs Ann. Chem. 653, 105-113 (1962). 
156. R. HUISGEN, J. SAUER, M. SEIDEL - Ringßffnungen der Azole, VIII. Zur Thermolyse des 5-Phenyl-tetrazols. Liebigs Ann. Chem. 654, 146-160 (1962).

157. R. HUISGEN, R. GRASHEY, R. KRISCHKE - 1,3-Additionen mit Pyridin-imin, Chinolin-imin, lsochinolin-imin und Thenanthridin-imin. Tetrahedron Lett. 387-391 (1962).

158. R. HUISGEN, W. EDL - Decarboxylierung von Diacylperoxyden zu Carbonestern. Angew. Chem. 74, 588 (1962); Angew. Chem., Int. Ed. Engl. 1, 458 (1962).

159. R. HUISGEN, R. GRASHEY, M. SEIDEL, H. KNUPFER, R. SCHMIDT - 1,3-Dipolare Additionen, 3. Umsetzungen des Diphenylnitrilimins mit Carbonyl- und Thiocarbonylverbindungen. Liebigs Ann. Chem. 658, 169-180 (1962).

160. R. HUISGEN - Synthesesvia 1,3-Dipolar Additions. Chem. Weekblad 59, 89-101 (1963).

161. R. HUISGEN, W.D. ZAHLER - Orientierungsphünomene bei der Substitution aromatischer Bicyclen, IV. Ionische und radikalische Mechanismen der intramolekularen Arylierung uber Diazoverbindungen. Chem. Ber. 96, 736-746 (1963).

162. R. HUISGEN, W.D. ZAHLER - Orientierungsphunomene bei der Substitution aromatischer Bicyclen, V. Weitere intramolekulare Arylierungen in der Naphthalin reihe und ihr Mechanismus. Chem. Ber. 96, 747-764 (1963).

163. W.D. ZAHLER, R. HUISGEN - Intramolekulare Arylierungen in der DiphenylmethanReihe. Chem. Ber. 96, 765-770 (1963).

164. R. HUISGEN - 1,3-Dipolare Cycloadditionen; RUckschau und Ausblick. Angew. Chem. 75, 604-637 (1963); Angew.Chem., Int. Ed. Engl. 2, 565-598 (1963).

165. R. HUIS GEN, R. KNORR - Sind Benz-ine verschiedener Provenienz identisch ? Tetrohedron Lett. 1017-1021 (1963).

166. R. HUISGEN - Kinetik und Mechanismus 1,3-Dipolarer Cycloadditionen. Angew. Chem. 75, 742-754 (1963); Angew. Chem., Int. Ed. Engl. 2, 633-645 (1963). 
167. R. HUISGEN, R. GRASHEY, E. STEINGRUBER - Azomethin-ylide und ihre 1,3Dipolaren Cycloodditionen. Tetrahedron Lett. 1963, 1441-1445 (1963).

168. R. HUISGEN, G. SEIDL - Mittlere Ringe, XX. Solvolyseprodukte der 1,2-Benzocyclenyl-(4)-tosylate. Chem. Ber. 96, 2730-2739 (1963).

169. R. HUISGEN, G. SEIDL - Mittlere Ringe, XXI. Ringerweiterungen bei der Solvolyse der 1, 2-Benzo- $\Delta^{\prime}$-cyclenyl-(3)-methyltosylate. Chem. Ber. 96, 2740-2749 (1963).

170. R. HUIS GEN, E. RAUENBUSCH, G. SEIDL, I. WIMMER - Mittlere Ringe, XIX. Kinetik der Solvolyse der 1,2-Benzocyclenyl-(4)-tosylate; ein Beitrag zur Beteiligung des $\beta$-stundigen Phenyls an der lonisation. Liebigs Ann. Chem. 671, 41-57 (1964).

171. G. SEIDL, R. HUISGEN - Mittlere Ringe, XXII. Die Reaktionen der 1,2-Benzocyclenyl-(4)-amine mit salpetriger Soure. Chem. Ber. 97, 249-254 (1964).

172. R. HUISGEN, H. SEIDL - 1,3-Additionen der Nitrone an Carbonester der Acetylenreihe. Tetrahedron Lett. 2019-2022 (1963).

173. H. SEIDL, R. HUISGEN - Umsetzungen des Isochinolin- und Phenanthridin-Noxids mit Carbonestern der Äthylen- und Acetylenreihe. Tetrahedron Lett. 20232025 (1963).

174. R. HUISGEN, F. MIETZSCH - Zur Valenztautomerie des Cyclooctatetraens. Angew. Chem. 76, 36-38 (1964); Angew. Chem., Int. Ed. Engl. 33, 83-85 (1964).

175. R. HUIS GEN, H. GOTTHARDT, H.O. BAYER - Azlactone als 1,3-Dipole. Eine Synthese von Pyrrolen. Angew. Chem. 76, 185 (1964); Angew.Chem., Int. Ed. Engl. $3,135-136$ (1964). 
176. R. HUISGEN, H. GOTTHARDT, H.O. BAYER, F.C. SCHAEFER - Eine neue Klasse mesoionischer Aromaten und ihre 1,3-Dipolaren Cycloadditionen mit Acetylenderivaten. Angew. Chem. 76, 185-186 (1964); Angew. Chem., Int. Ed.Engl. 3, 136-137 (1964).

177. R. HUISGEN, H. GOTTHARDT, H.O. BAYER - 1,3-Dipolare Cycloadditionen der Azlactone an CC-Doppelbindungen. Tetrahedron Lett. 481-485 (1964).

178. H. GOTTHARDT, R. HUISGEN, F.C. SCHAEFER - $\Delta^{2}-$ Pyrroline aus mesoionischen Oxazolen und Olefinen. Tetrahedron Lett. 487-491 (1964).

179. R. HUISGEN, G. SEIDL - Mittlere Ringe, XXIII. Solvolysen des 1,2-Benzo-cyclooctenyl-(3)-methyltosylats; Umlagerung des 1,2-Benzo-cyclononenyl-(4)-carboniumions. Tetrahedron 20, 231-241 (1964).

180. R. HUISGEN, G. SEIDL, I. WIMMER - Mittlere Ringe, XXIV. Kinetik der Solvolyse der 1,2-Benzo-cyclenyl-(3)-methyltosylate. Tetrahedron 20, 623-631 (1964).

181. G. SEIDL, R. HUISGEN, J.H.M. HILL - Mittlere Ringe, XXV. Umsetzungen der 1,2-Benzocyclenyl-(3)-methylamine mit salpetriger Säure. Tetrahedron 20, 633640 (1964).

182. R. HUISGEN, R. GRASHEY, H. KNUPFER, R. KUNZ, M. SEIDEL - 1,3-Dipolare Cycloadditionen, 6. Anlagerung der Nitrilimine an Azomethine und isocyanate. Chem. Ber. 97, 1085-1095 (1964).

183. R. HUISGEN, H. BLASCHKE - 1,3-Cycloadditionen des Äthoxycarbonyl-azens an Alkine. Tetrahedron Lett. 1409-1413 (1964).

184. R. HUISGEN - Kinetik und Mechanismus 1,3-Dipolarer Cycloadditionen. DechemaMonographien 49, 201-222 (1964), Verlag Chemie, Weinheim/Bergstraße.

185. R. HUISGEN, G. BINSCH, L. GHOSEZ - 1,3-Dipolare Cycloadditionen, 7. Abfangen der Ketocarben-Zwischenstufe bei der Thermolyse von Diazoketonen. Chem. Ber. 97, 2628-2639 (1964). 
186. R. HUISGEN, H.J. STURM, G. BINSCH - 1,3-Dipolare Cycloadditionen, 8. 1,3-Additionen des Äthoxycarbonyl-carbens an Nitrile. Chem. Ber. 97, 2864-2867 (1964).

187. R. HUISGEN, G. BINSCH, H. KÖNIG - 1,3-Dipolare Cycloodditionen, 9. Umsetzungen aromatischer Ketocamene mit Heteromehrfachbindungen. Chem. Ber. 97, $2864-2883$ (1964).

188. R. HUISGEN, G. BINSCH, H. KÖNIG - 1,3-Dipolare Cycloadditionen, 10. Thermolysen aromatischer o-Diazo-oxide in Alkinen und Aromaten. Chem. Ber. 97, 2884-2892 (1964).

189. G. BINSCH, R. HUISGEN, H. KÖNIG - 1,3-Dipolare Cycloadditionen, 11. Die Anlagerung des aromatischen Ketocarbens aus 3,4,5,6-Tetrachlor-benzol-2diazo-1-oxid an Alkene. Chem. Ber. 97, 2893-2902 (1964).

190. R. HUISGEN, G. SEIDL, 1. WIMMER - Mittlere Ringe, XXVI. Sterische Mesomeriehinderung in Methoxy-substituierten 1,2-Benzo-cyclenonen-(3). Liebigs Ann. Chem. 677, $21-33$ (1964).

191. G. SEIDL, R. HUISGEN, I. WIMMER - Mittlere Ringe, XXVII. Die Beteiligung methoxylierten $\beta$ - thenyis an der Ionisation; Versuche zur Isolierung verbruckter Zwischenstufen. Liebigs Ann. Chem. 677, 34-46 (1964).

192. R. HUISGEN, H. SEIDL - Die Valenztautomerie des 1,2-Diphenyl-benzocyclobutens. Tetrahedron Lett. 3381-3386 (1964).

193. R. HUISGEN, L. FEILER, G. BINSCH - Stereospezifische Addition von Ketenen an Enoluther. Angew. Chem. 76, 892-893 (1964); Angew.Chem., Int. Ed. Engl. 3 , 753-754 (1964).

194. R. HUISGEN, R. GRASHEY, J. SAUER - Cycloaddition Reactions of Alkenes, in "The chemistry of alkenes" (Herausgeber S. Patai), S. 739-953, Interscience Publ., London - New York, 1964.

195. R. HUISGEN - 1,3-Dipolar Additions. Proc. Robert A. Welch Foundation Conferences on Chemical Research, IV. Molecular Structure and Organic Reactions (1961); 60-95 (1964). 
196. R. GRASHEY, R. HUISGEN, K.K. SUN, R.M. MORIARTY - 1,3-Dipolar Cycloadditions, 12. The Synthesis of 1,3,4-Thiadiazolidine-5-thiones. J. Org. Chem. 30, 74-79 (1965).

197. R. HUISGEN, R. GRASHEY, E. AUFDERHAAR, R. KUNZ - 1,3-Dipolare Cycloadditionen, 13. Additionen der Nitrilimine on Oxime, Azine und andere CN-Doppelbindungen. Chem. Ber. 98, $642-649$ (1965).

198. R. HUISGEN, W. KOLBECK - N-Acyloxy-ammonium-Salze. Tetrahedron Lett. $783-787$ (1965).

199. R. HUISGEN, L. MÖBIUS, G. SZEIMIES - 1,3-Dipolare Cycloadditionen, 14. Die Anlagerung organischer Azide an Enoläther: Orientierung und Triazolin-Zerfall. Chem. Ber. 98, 1138-1152 (1965).

200. R. HUISGEN, G. SZEIMIES - 1,3-Qipolare Cycloadditionen, 15. Der sterische Ablauf der Azid-Addition an Enolather. Chem. Ber. 98, 1153-1158 (1965).

201. R. HUISGEN, E. AUFDERHAAR, G. WALLBILLICH - 1,3-Dipolare Cycloadditionen, 16. Zur Bildung von 1,4-Dihydro-tetrazinen aus Nitriliminen; 1,4-Dipheny1-1,4-dihydro-1, 2,4,5-tetrazin und isomere Verbindungen. Chem. Ber. 98, 1476-1486 (1965).

202. R. HUISGEN, G. BOCHE - Zum Mechanismus der Bromierung des Cyclooctatetraens. Tetrahedron Lett. 1769-1774 (1965).

203. G. BOCHE, R. HUISGEN - Polybromide des Cyclooctatetraens. Tetrahedron Lett. 1775-1777 (1965).

204. R. HUISGEN, R. GRASHEY, R. KUNZ, G. WALLBILLICH, E. AUFDERHAAR 1,3-Dipolare Cycloadditionen, 17. Bisaddukte aus Nitriliminen und Carbodiimiden. Chem. Ber. $98,2174-2184$ (1965).

205. R. HUISGEN, E. AUFDERHAAR - 1,3-Dipolare Cycloadditionen; 18. Die Umsetzung des Phenyl-cyanamids mit $\mathrm{C}$ - $\ddot{A}$ thoxycarbonyl-N-phenyl-nitrilimin. Chem. Ber. 98, 2185-2192 (1965).

206. R. HUISGEN, F. MIETZSCH, G. BOCHE, H. SEIDL - The Valence Tautomerism of Unsaturated Ring Systems. Special Rubl. No.19, 3-21, Chemical Society, London 1965. 
207. R. HUISGN, H. BLASCHKE - 1,3-Dipolare Cycloadditionen, 19. Zur Addition des Äthoxycarbonyl-azens on Nitrile. Liebigs Ann.Chem. 686, 145-153 (1965).

208. R. HUIS GEN, H. BLASCHKE - 1,3-Dipolare Cycloadditionen, 20. 2-Äthoxyoxazole aus Äthoxycarbonyl-azen und Alkinen. Chem. Ber. $98,2985-2997$ (1965).

209. R. HUISGEN, J.P. ANSELME - 1,3-Dipolare Cycloadditionen, 21. Abfangen der Ketoazen-Zwischenstufe bei der Photolyse der Acylazide. Chem. Ber. 98, 29983000 (1965).

210. R. HUISGEN, C. AXEN, H. SEIDL - Ringöffnungen der Azole, 1X. Synthese oligomerer 1,3,4-Oxadiazolyl-m-phenyle. Chem. Ber. 98, 2966-2984 (1965).

211. R. HUISGEN, K. HERBIG - 1,4-Dipolare Cycloadditionen, I. Reaktionen der Azomethine mit Acetylendicarbonsdureester. Liebigs Ann. Chem. 688, 98-112 (1965).

212. R. HUISGEN, L. MÖBIUS, G. MÜLLER, H. STANGL, G. SZEIMIES, J.M. VERNON. 1,3-Dipolare Cycloadditionen, 22. Zur Anlogerung organischer Azide an winkelgespannte Doppelbindungen. Chem. Ber. 98, 3992-4013 (1965).

213. R. HUISGEN, R. KNORR, L. MÖBIUS, G. SZEIMIES - 1,3-Dipolare Cycloadditionen, 23. Einige Beobachtungen zur Addition organischer Azide an CC-Dreifachbindungen. Chem. Ber. 98, 4014-4021 (1965).

214. R. HUISGEN, R. GRASHEY, J.M. VERNON, R. KUNZ - Umsetzungen von $\Delta^{2}-$ Triazolinen und von Ringketon-Anilen mit Isocyanaten und Isothiocyanaten. Tetrahedron $21,3311-3325$ (1965).

215. R. HUISGEN - Cycloaddition par Dipoles-1,3. Rétrospective et Progrès. Bull. Soc. Chim. France, 3431-3440 (1965).

216. R. HUISGEN - Cycloadditionen. Chemie in Labor und Betrieb 17, 1-8, 57-66 (1966).

217. R. HUISGEN, W. SCHEER, G. SZEIMIES, H. HUBER - 1,3-Cycloadditionen von Azomethin-yliden aus Aziridin-carbonestern. Tetrahedron Lett. 397-404 (1966). 
218. R. HUISGEN, H. BLASCHKE, E. BRUNN - Zur 1,3-Dipolaren Cycloaddition aromatischer Nitriloxide an Azodicarbonstureester. Tetrahedron Lett. 405-409 (1966).

219. R. HUISGEN, R. RAAB - Neve 1,3-Dipolare Cycloodditionen der Nitrilylide und eine Tautomerie bei Imidchloriden. Tetrahedron Lett. 649-654 (1966).

220. R. HUISGEN, G. SZEIMIES, L. MÖBIUS - 1,3-Dipolare Cycloadditionen, 24. Triazoline aus organischen Aziden und $\alpha, \beta$-ungesuttigten Carbonylverbindungen oder Nitrilen. Chem. Ber. 99, 475-490 (1966).

221. G. SZEIMIES, R. HUISGEN - Themolyse der $\Delta^{2}-1,2,3$-Triazolin-4-carbonester; Chemismen des Triazolin-Zerfalls. Chem. Ber. 99, 491-503 (1966).

222. R. HUISGEN, G. BOCHE, W. HECHTL, H. HUBER - Zur Chlorierung des Cyclooctatetraens. Angew. Chem. 78, 595 (1966); Angew. Chem., Int. Ed. Engl. 5, 585586 (1966).

223. R. HUISGEN, R. SUSTMANN, K. BUNGE - Geht der 1,3-Dipolaren Cycloaddition eine 1, 1-Addition voraus? Tetrahedron Lett. 3603-3610 (1966).

224. R. HUISGEN, K. HERBIG, A. SIEGL, H. HUBER - Die Addukte primärér und sekundurer Amine an Carbonester der Acetylenreihe und ihre Konfiguration. Chem. Ber. 99, 2526-2545 (1966).

225. K. HERBIG, R. HUISGEN, H. HUBER - cis-trans-isomerisierung bei Enamin- $\beta$-carbonestern; zum sterischen Ablauf der Amin-Addition on Carbonester der Acetylenreihe. Chem. Ber. $99,2546-2555$ (1966).

226. J.S. CLOVIS, A. ECKELL, R. HUISGEN, R. SUSTMANN - 1,3-Dipolare Cycloadditionen, 25. Der Nachwe is des freien Diphenylnitrilimins als Zwischenstufe bei Cycloadditionen. Chem. Ber. 100, 60-70 (1967).

227. R. HUISGEN, V. WEBERNDÖRFER - 1,3-Dipolare Cycloodditionen, 26. Intramolekulare Stabilisierung bei einem N-Trinitrophenyl-nitrilimin. Chem. Ber. 100, 71-78 (1967).

228. R. HUISGEN - Ist die Heterocyclische Chemie langweilig ? Nachrichten aus Chemie und Technik, 487 (1966). 
229. R. HUISGEN, J. WULFF - Cycloadditionen von Nitriloxiden an Methylenphosphorane. Tetrahedron Lett. 917-920 (1967).

230. R. HUISGEN, J. WULFF - Umsetzungen der Nitriloxide mit Iminophosphoranen. Tetrohedron Lett. $921-923$ (1967).

231. R. HUISGEN, M. MORIKAWA, K. HERBIG, E. BRUNN - 1,4-Dipolare Cycloodditionen, II. Dreikomponenten-Reaktionen des isochinolins mit Acetylendicarbonsdureester und verschiedenen Dipolarophilen. Chem. Ber. 100, 1094-1106 (1967).

232. R. HUIS GEN, K. HERBIG, M. MORIKAWA - 1,4-Dipolare Cycloadditionen, III. Umsetzungen des Isochinolins und der Azomethine mit Phenylisocyanat. Chem. Ber. $100,1107-1115(1967)$.

233. R. HUISGEN, E. FUNKE - 1,3-Cycloadditionen mesoionischer Oxazolone an Carbonylverbindungen. Angew. Chem. 79, 320 (1967); Angew. Chem., Int. Ed. Engl. 6, 365-366 (1967).

234. R. HUIS GEN, E. FUNKE, F.C. SCHAEFER, R. KNORR - Valenztautomerie eines mesoionischen Oxazol-5-ons mit einem Acylamino-keten? Angew. Chem. 79, 321 〈1967); Angew. Chem., Int. Ed.Engl. 6, 367 (1967).

235. R. HUISGEN, E. FUNKE, F.C. SCHAEFER, H. GOTTHARDT, E. BRUNN - Cycloadditionen mesoionischer Oxazol-5-one an einige Heteromehrfachbindungen. Tetrahedron Lett. 1809-1814 (1967).

236. R. HUISGEN, W. SCHEER, H. HUBER - Stereospecific Conversion of cis-trans isomeric Aziridines to Open-Chain Azomethine Ylides. J.Am.Chem. Soc. 89, 1753-1755 (1967).

237. R. HUISGEN, H. KNUPFER, R. SUSTMANN, G. WALLBILLICH, V. WEBERNDÖRFER - 1,3-Dipolare Cycloadditionen, 27. Zur Anlagerung des Dipheny Initrilimins an nichtkonjugierte Alkene und Alkine; Sterischer Ablauf, Orientierung und Substituenteneinfluß. Chem. Ber. 100, 1580-1592 (1967).

238. J.S. CLOVIS, A. ECKELL, R. HUISGEN, R. SUSTMANN, G. WALLBILLICH, V. WEBERNDÖRFER - 1,3-Dipolare Cycloodditionen, 28. Diphenylnitrilimin und arylkonjugierte Alkene. Chem. Ber. 100, 1593-1601 (1967). 
239. R. HUISGEN, M. MORIKAWA, D.S. BRESLOW, R. GRASHEY - 1,4-Dipolare Cycloadditionen, IV. Reaktionen der Azomethine mit lsothiocyanaten und Schwefelkohlenstoff. Chem. Ber. 100, 1602-1615 (1967).

240. M. MORIKAWA, R. HUIS GEN - 1,4-Dipolare Cycloadditionen, V. Einige Umsetzungen des 3,4-Dihydro-isochinolins mit elektrophilen CC-Mehrfachbindungen. Chem. Ber. 100, 1616-1620 (1967).

241. R. HUISGEN, B. GIESE, H. HUBER - Neves zum sterischen Ablauf der Amin-Addition an Carbonester der Acetylenreihe. Tetrahedron Lett. 1883-1888 (1967).

242. B. GIESE, R. HUISGEN - Zur Kinetik der Amin-Addition an Carbonester der Acetylenreihe. Tetrahedron Lett. 1889-1892 (1967).

243. R. HUISGEN, M. CHRISTL - 1,3-Dipolare Cycloodditionen der Knallsdure. Angew. Chem. 79, $471-472$ (1967); Angew. Chem., Int. Ed. Engl. 6, 456-457 (1967).

244. J. WULFF, R. HUISGEN - 1,3-Cycloadditionen der Nitrone an Methylen-phosphorane. Angew. Chem. 79, 472 (1967); Angew. Chem., Int. Ed. Engl. $\underline{6}, 457-458$ (1967).

245. R. HUISGEN, R. SUSTMANN, G. WALLBILLICH - 1,3-Dipolare Cycloadditionen, 29. Orientierungsphünomene bei der Anlagerung von Nitriliminen an $a, \beta$-ungesüttigte Carbonester, Vinylüther und Enamine. Chem. Ber. 100, 1786-1801 (1967).

246. R. SUSTMANN, R. HUISGEN, H. HUBER - 1,3-Dipolare Cycloadditionen, 30. Substituenteneffekte in den Kernresonanzspektren von 1,3-Diphenyl- $\Delta^{2}$-pyrazolinen und 3-Phenyl- $\Delta^{2}$-isoxazolinen. Chem. Ber. 100, 1802-1813 (1967).

247. A. ECKELL, R. HUISGEN, R. SUSTMANN, G. WALLBILLICH, D. GRASHEY, E. SPINDLER - 1,3-Dipolare Cycloadditionen, 31. Dipolarophilen-Aktivitaten gegenUber Diphenylnitrilimin und zahlenmaßige Ermittlung der Substituenteneinflusse. Chem. Ber. 100, 2192-2213 (1967).

248. G. BOCHE, W. HECHTL, H. HUBER, R. HUISGEN - endo- and exo-8-Chlorohomotropylium Salts. J. Amer. Chem. Soc. 89, 3344-3345 (1967). 
249. R. HUISGEN, G. BOCHE, H. HUBER - The Halogenation of Cyclooctatetraene via 8-Holohomotropylium lons. J.Am. Chem. Soc. 89, 3345-3346 (1967).

250. R. HUISGEN, G. SZEIMIES, L. MOBBIUS - 1,3-Dipolare Cycloadditionen, 32. Kinetik der Additionen organischer Azide an CC-Mehrfachbindungen. Chem. Ber. $100,2494-2507$ (1967).

251. R. HUISGEN - Cycloaddition Reactions of Mesoionic Aromatic Compounds. Special Publ. Nr. 21, 51-73, Chemical Society, London 1967.

252. R. HUISGEN, K. ADELSBERGER, E. AUFDERHAAR, H. KNUPFER, G. WALLBILLICH - 1,3-Dipolare Cycloadditionen 33. Unterschiedliche Reaktivitdten substituierter Nitrilimine. Monatsh. Chem. 98, 1618-1650 (1967).

253. R. HUISGEN, A. DAHMEN, H. HUBER - Stereospecific Conrotatory Volence Isomerization of Octatetraenes to Cycloocta-1.3.5-trienes. J.Am. Chem. Soc. 89, 71307131 (1967).

254. R. HUISGEN - Neues Uber 1,3-Cycloadditionen. Helv. Chim. Acta 50, 24212439 (1967).

255. R. HUISGEN, H. GOTTHARDT, R. GRASHEY - 1,3-Dipolare Cycloadditionen, 34 . Ryrazole aus Sydnonen und acetylenischen Dipolarophilen. Chem. Ber. 101, 536551 (1968).

256. H. GOTTHARDT, R. HUISGEN - 1,3-Dipolare Cycloadditionen, 35. $\triangle^{2}$-Pyrazoline aus Sydnonen und Alkenen. Chem. Ber. 101, 552-563 (1968).

257. R. HUIS GEN, R. GRASHEY, H. GOTTHARDT - 1,3-Dipolare Cycloadditionen, 36. Pyrazole aus Sydnonen und $a, \beta$-ungestttigten Nitrilen oder Carbonylverbindungen. Chem. Ber. 101, 829-838 (1968).

258. R. HUISGEN, H. GOTTHARDT - 1,3-Dipolare Cycloadditionen, 37. Pyrazole aus Sydnonen und 1,1-disubstituierten Äthylenen unter Kohlenwasserst off-Abspaltung und verwandte Reaktionen. Chem. Ber. 101, 839-846 (1968). 
259. H. GOTTHARDT, R. HUISGEN, R. KNORR - 1,3-Dipolore Cyçloadditionen, 38. Reaktionen der Sydnone mit Benz-in und mit einigen Heteromehrfachbindungen. Chem. Ber. 101, 1056-1058 (1968).

260. R. HUISGEN, H. GOTTHARDT - 1,3-Dipolare Cycloodditionen, 39. Kinetik und Mechanismus der Cycloadditionen der Sydnone. Chem. Ber. 101, 1059-1071 (1968).

261. R. HUISGEN - Cycloadditionen - Begriff, Einteilung und Kennzeichnung. Angew. Chem. 80, 329-372 (1968); Angew. Chem., Int. Ed. Engl. 7, 321-328 (1968).

262. R. HUISGEN, R. GRASHEY, H. HAUCK, H. SEIDL - 1,3-Dipolare Cycloadditionen, 40. Isoxazolidine aus Nitronen und gewöhnlichen oder winkelgespannten Alkenen. Chem. Ber. 101, 2043-2055 (1968).

263. R. HUISGEN - On the Mechanism of 1,3-Dipolar Cycloadditions: A Reply. J. Org. Chem. 33, 2291-2297 (1968).

264. R. HUISGEN, R. GRASHEY, H. HAUCK, H. SEIDL - 1,3-Dipolare Cycloadditionen, 41. Anlagerung der Nitrone an Styrol; Orientierung und rodumlicher Ablauf. Chem. Ber. 101, 2548-2558 (1968).

265. R. HUISGEN, R. GRASHEY, H. SEIDL, H. HAUCK - 1,3-Dipolare Cycloadditionen, 42. Additionen der Nitrone an weitere arylkonjugierte Äthylene sowie an Vinylather. Chem. Ber. 101, 2559-2567 (1968).

266. R. HUISGEN, H. HAUCK, R. GRASHEY, H. SEIDL - 1,3-Dipolare Cycloadditionen, 43. Isoxazolidine aus Nitronen mit a, $\beta$-ungesättigten Carbonestem oder Nitrilen. Chem.Ber. 101, 2568-2584 (1968).

267. R. HUIS GEN - Synthese von Heterocyclen mit 1,4-Dipolaren Cycloadditionen. Z. Chem. $\underline{8}, 290-320$ (1968).

268. R. HUISGEN, L. A. FEILER, P. OTTO - Zur Kinetik der Cycloadditionen des Diphenylketens. Tetrahedron Lett. 4485-4490 (1968).

269. R. HUISGEN, P. OTTO - Cycloodditionen des Diphenylketens an 1,3-Diene. Tetrohedron Lett. $4491-4495$ (1968). 
270. G. BINSCH, L. A. FEILER, R. HUISGEN - Sterischer Ablauf und Mechanismus der Cycloadditionen der Ketene an CC-Doppelbindungen. Tetrahedron Lett. 4497-4501 (1968).

271. P. OTTO, L.A. FEILER, R. HUISGEN - Cyclobutanone aus Enaminen und Ketenen in zweistufiger Addition. Angew. Chem. 80, 759-760 (1968); Angew. Chem., Int. Ed. Engl. 9, 737-738 (1968).

272. R. HUISGEN, B.A. DAVIS, M. MORIKAWA - Nachweis einer offenkettigen Zwischenstufe bei der $\beta$-Lactam-Bildung aus Ketenen und Azomethinen. Angew. Chem. 80, 802-803 (1968); Angew. Chem., Int. Ed. Engl. 10, 826 (1968).

273. R. HUISGEN, P. OTTO - The Mechanism of Dimerization of Dimethylketene. J. Am. Chem. Soc. 90, 5342-5343 (1968).

274. M. CHRISTL, R. HUISGEN - Alte und neve Cycloadditionen der Nitriloxide. Tetrahedron Lett. 5209-5213 (1968).

275. R. HUISGEN, G. BOCHE, A. DAHMEN, W. HECHTL - Substituenteneinflusse auf das Tautomerie-Gleichgewicht Cycloocta-1,3,5-trien / Bicyclo(4.2.0)octadien. Tetrahedron Lett. 5215-5219 (1968).

276. R. HUISGEN, H. HAUCK, R. GRASHEY, H. SEIDL - 1,3-Dipolare Cycloadditionen, 44. Nitrone und $\alpha, \beta$-ungesättigte Dicarbonylverbindungen; Stereospezifität der Cycloadditionen. Chem. Ber. 102, 736-745 (1969).

277. R. HUISGEN, J. WULFF - 1,3-Dipolare Cycloadditionen, 45. Additionen der Nitrone an Methylenphosphorane. Chem. Ber. 102, 746-754 (1969).

278. H. SEIDL, R. HUISGEN, R. KNORR - 1,3-Dipolare Cycloadditionen, 46. Zur Anlagerung der Nitrone an CC-Dreifochbindungen. Chem. Ber. 102, $904-914$ (1969).

279. R. HUISGEN, H. SEIDL, J. WULFF - 1,3-Dipolare Cycloadditionen, 47. Reaktionen heteroaromatischer Aminoxide mit Carbonestern der Äthylen- und Acetylenreihe. Chem. Ber. 102, 915-925 (1969). 
280. H. SEIDL, R. HUISGEN, R. GRASHEY - 1,3-Dipolore Cycloodditionen, 48. Einige Umsetzungen von Nitronen und heteroaromatischen Aminoxiden mit Phenylisocyanat und Phenyisenfol. Chem. Ber. 102, 926-930 (1969).

281. R. HUISGEN, H. SEIDL, I. BRUNNING - 1,3-Dipolare Cycloodditionen, 49. Kinetik und Mechanismus der Nitron-Additionen an ungesattigte Verbindungen. Chem.Ber. 102, 1102-1116 (1969).

282. R. HUISGEN, H. HAUCK, H. SEIDL, M. BURGER - 1,3-Dipolore Cycloadditionen, 50. Charakterisierung ungesdttigter Verbindungen als Acyl-nitron-Addukte. Chem. Ber. 102, $1117-1128$ (1969).

283. R. HUISGEN, A. DAHMEN, H. HUBER - ZUr Kinetik der conrotatorischen Valenzisomerisierung von stereoisomeren Decatetraenen. Tetrahedron Lett. 1461-1464 (1969).

284. A. DAHMEN, R. HUISGEN - Zur Stereospezifitut der conrotatorischen Cyclisierung des Decatetraens. Tetrahedron Lett. 1465-1469 (1969).

285. R. HUISGEN - Synthesis via 1,4-Dipolar Cycloadditions. X. Corso Estivo di Chimica: Moderni Sviluppi della Sintesi Organica. Accad. Naz. dei Lincei (Rom), 239257 (1968).

286. R. HUISGEN - Nitrile Ylides and Azomethine Ylides, New Classes of Compounds. $X$. Corso Estivo di Chimica: Moderni Sviluppi della Sintesi Organica. Accad. Naz. dei Lincei (Rom), 259-280 (1968).

287. R. HUISGEN - Reactions and Rearrangements in the Cyclooctatetraene Series. X. Corso Estivo di Chimica: Moderni Sviluppi della Sintesi Organica. Accad. Naz. dei Lincei (Rom), 281-301 (1968).

288. R. HUISGEN, J. WULFF - 1,3-Dipolare Cycloadditionen, 51. Die Anlagerung der Nitriloxide on Methylenphosphorane. Chem. Ber. 102, 1833-1840 (1969).

289. J. WULFF, R. HUISGEN - Reaktionen der Methylenphosphorane mit weiteren 1,3Dipolen sowie mit Styroloxid. Chem.Ber. 102, 1841-1847 (1969). 
290. R. HUIS GEN, J. WULFF - 1,3-Dipolare Cycloadditionen, 52. Umsetzungen von 1,3-Dipolen mit Iminophosphoranen. Chem.Ber. 102, 1848-1858 (1969).

291. E. BRUNN, R. HUISGEN - Struktur und Reaktivitut des Betains aus Triphenyl-phosphin und Azodicarbonsäureester. Angew. Chem. 81, 534-536 (1969); Angew. Chem., Int. Ed. Engl. 8, 513-51 5 (1969).

292. R. HUISGEN, K.v.FRAUNBERG, H.J. STURM - Cycloadditionen von Ryridyl-und Pyrimidyl-aziden. Tetrahedron Lett. 2589-2594 (1969).

293. R. HUISGEN, K.v.FRAUNBERG - Zur Thermolyse des 2-Azido-4,6-dimethyl-pyrimidins; Reaktionen des Azens. Tetrahedron Lett. 2595-2598 (1969).

294. K.V.FRAUNBERG, R. HUISGEN - Kupferkatalysierte Zerfal lsreaktionen von Imidaziden; Reaktionen von Kupferazenen. Tetrahedron Lett. 2599-2602 (1969).

295. R. HUISGEN, W. SCHEER, H. MÄDER - Azomethin-ylide aus Aziridin-dicarbonsäureestem: Kinetik der cis-trans-lsomerisierung und der Ringöffnung. Angew. Chem. 81, 619-620 (1969); Angew. Chem., Int. Ed. Engl. 8, 602-604 (1969).

296. R. HUISGEN, W. SCHEER, H. MÄDER, E. BRUNN - Unterschiedliche 1,3-dipolare Aktivität cis, trans-isomerer Azomethin-yliddicarbonsöureester. Angew. Chem. 81, 621 (1969); Angew. Chem., Int. Ed. Engl. 8, 604 (1969).

297. R. HUISGEN, H. MÄDER - Zur Konfigurotion eines cis-disubstituierten Azomethinylids. Angew. Chem. 81, 621-623 (1969); Angew. Chem., Int. Ed.Engl. 8, 604606 (1969).

298. R. HUISGEN, L.A. FEILER - Cycloodditionen der Ketene, I. Diphenylketen und gewöhnliche Alkene. Chem. Ber. 102, 3391-3404 (1969).

299. R. HUISGEN, L. A. FEILER, P. OTTO - Cycloadditionen der Ketene, II. Umsetzungen des Diphenylketens mit Vinylathem. Chem. Ber. 102, 3405-3427 (1969).

300. L.A. FEILER, R. HUISGEN - Cycloodditionen der Ketene, III. Diphenylketen und einige Enamine. Chem. Ber. 102, 3428-3443 (1969). 
301. R. HUISGEN, L.A. FEILER, P. OTTO - Cycloadditionen der Ketene, IV. Kinetik der Cyclobutanon-Bildung aus Diphenyl keten und ungesdttigten Verbindungen. Chem. Ber. 102, 3444-3459 (1969).

302. R. HUISGEN, L. A. FEILER, G. BINSCH - Cycloadditionen der Ketene, V. Stereospezifische Cyclobutanon-Bildung ous Ketenen mit cis,trans-isomeren Vinylathern. Chem.Ber. 102, 3460-3474 (1969).

303. R. HUISGEN, P. OTTO - Cycloadditionen der Ketene, VI. Zur Anlagerung des Diphenylketens an 1,3-Diene. Chem. Ber. 102, 3475-3485 (1969).

304. R. HUISGEN - 1,4-Dipolar Cycloaddition: A General Principle of Heterocyclic Synthesis, in "Topics in Heterocyclic Chemistry" (Herausg. R.N. Castle), S. 223-252, Wiley - Interscience, 1969.

305. R. HUISGEN, P. OTTO - Demonstration of Two Mechanistic Pathways in the Reaction of Dimethylketene with $\mathrm{N}$-Isobutenylpyrrolidine. J. Am. Chem. Soc. 91, 59225923 (1969).

306. R. HUIS GEN - Electrocyclic Reactions and Orbital Symmetry Control. Lo Chimica e L'Industria 51, 963-970 (1969).

307. R. HUISGEN, E. BRUNN, R. GIRARDI, I. KARLE - Structure and Cycloaddition of a Quasi 1,3 Dipole from Triphenylphosphine and 4-Chlorobenzene Diazocyanide. J.Am.Chem. Soc. 91, 7766-7767 (1969).

308. R. HUISGEN, W.E. KONZ - Kinetics and Mechanism of the Rearrangement of Bromocyclooctatetraene to trans- $\beta$-Bromostyrene. J. Am. Chem. Soc. 92, 4102-4104 (1970).

309. W.E. KONZ, W. HECHTL, R. HUISGEN - Further Contributions to the Mechanism of the Halocyclooctatetraene Rearrangement. J.Am. Chem. Soc. 92, 4104-4105 (1970).

310. R. HUISGEN, W.E. KONZ, G.E. GREAM - Evidence for Different Valence Tautomers of Bromocyclooctotetraene. J.Am. Chem. Soc. 92, 4105-4106 (1970). 
311. H. GOTTHARDT, R. HUISGEN, O. BAYER - 1,3-Dipolar Cycloaddition Reactions, 53. The Question of the 1,3-Dipolar Nature of $\Delta^{2}$-Oxazolin-5-ones. J.Am. Chem. Soc. $92,4340-4344$ (1970).

312. H.O. BAYER, H. GOTTHARDT, R. HUISGEN - 1,3-Dipolare Cycloadditionen, 54. Byrrole aus Azlactonen und Acetylencarbonestem. Chem. Ber. 103, 2356-2367 (1970).

313. R. HUISGEN, H. GOTTHARDT, H.O. BAYER - 1,3-Dipolare Cycloadditionen, 55. $\Delta^{\prime}$-Ryrroline und 7-Azabicyclo(2.2.1)heptane aus Azlactonen und aktivierten Alkenen. Chem. Ber. 103, 2368-2387 (1970).

314. H.O. BAYER, R. HUISGEN, R. KNORR, F.C. SCHAEFER - Darstellung und Eigenschaften mesoionischer Oxazolone. Chem. Ber. 103, 2581-2597 (1970).

315. R. KNORR, R. HUISGEN - Zum Mechanismus der Dakin-West-Reaktion, I: Die Reaktion von N-Acyl-sek.-aminosouren mit Acetanhydrid. Chem. Ber. 103, 2598$2610(1970)$.

316. R. HUISGEN, H. GOTTHARDT, H.O. BAYER, F.C. SCHAEFER - 1,3-Dipolare Cycloadditionen, 56. Eine bequeme Synthese von $\mathrm{N}$-substituierten Ryrrolen aus mesoionischen Oxazolonen und Alkinen. Chem. Ber. 103, 2611-2624 (1970).

317. H. GOTTHARDT, R. HUISGEN - 1,3-Dipolare Cycloadditionen, 57. $\Delta^{2}$-Pyrroline aus $\mathrm{N}$-substituierten Oxazolium-5-oxiden und olefinischen Dipolarophilen. Chem. Ber. 103, 2625-2638 (1970).

318. R. KNORR, R. HUISGEN, G.K. STAUDINGER - 1,3-Dipolare Cycloadditionen, 58. Kinetik der Cycloadditionen eines Oxazolium-5-oxids. Chem. Ber. 103, 2639-2646 (1970).

319. U. BURGER, R. HUISGEN - Cyclopropanes from Olefins and Lithium Carbenoids. Tet rahedron Lett. 3049-3051 (1970).

320. R. HUISGEN, U. BURGER - Are Different Lithium Carbenoids Responsible for Homologation and Methylenation? Tetrahedron Lett. 3053-3056 (1970).

321. U. BURGER, R. HUISGEN - Competition of Alkenes for Metal Carbenoids. Tetrahedron Lett. $3057-3060$ (1970). 
322. R. HUISGEN - Zum kinetischen Nachweis reaktiver Zwischenstufen. Angew. Chem. 82, 783-794 (1970); Angew. Chem., Int. Ed. Engl. ㅁ, 751-762 (1970).

323. R: HUISGEN - Saul Winstein, 8.10.1912 - 23.11.1969 (Nachruf). Bayer. Akademie der Wissenschaften, Jahrbuch 1970, S. 232-238, Munchen.

324. R. HUISGEN, V. MARTIN-RAMOS, W. SCHEER - Cycloadditions of an Aziridine via Azomethine Ylide to Heteromultiple Bonds. Tetrahedron Lett. 477-480 (1971).

325. E. BRUNN, R. HUISGEN - Cycloodditions of Aziridines to Azo Compounds. Tetrohedron Lett. 473-476 (1971).

326. R. HUISGEN, W. SCHEER - Dipolarophilic Activity of Aromatic Bonds Towards an Azomethine Ylide. Tetrahedron Lett. 481-484 (1971).

327. R. HUISGEN, H. MÄDER - Azomethine Ylide from Dimethyl 1-(p-Methoxyphenyl) aziridine-2,3(cis)-dicarboxylate. Kinetics of the Thermal Ring Opening. J.Amer. Chem. Soc. 93, 1777-1779 (1971).

328. H. HERMANN, R. HUISGEN, H. MÄDER - Azomethine Ylides by Photolysis of Isomeric Dimethyl 1-(p-Methoxyphenyl)aziridine-2,3-dicarboxylates. Elaboration of the Total Energy Profile. J.Am.Chem.Soc. 93, 1779-1780 (1971).

329. R. HUIS GEN, E. FUNKE, H. GOTTHARDT, H. L. PANKE - 1,3-Dipolare Cycloadditionen, 59. Umsetzungen mesoionischer Oxazolone mit Carbonylverbindungen. Chem. Ber. 104, 1532-1549 (1971).

330. E. FUNKE, R. HUISGEN, F.C. SCHAEFER - 1,3-Dipolare Cycloodditionen, 60. Cycloadditionen $\mathrm{N}$-substituierter Oxazolium-5-olate an Thiocarbonyl-Verbindungen. Chem.Ber. 104, 1550-1561 (1971).

331. E. 8RUNN, E. FUNKE, H. GOTTHARDT, R. HUISGEN - 1,3-Dipolare Cycloadditionen, 61. Cycloadditionen von $\mathrm{N}$-substituierten Oxazolium-5-olaten an Nitrile, Nitro-, Nitroso- und Azoverbindungen. Chem. Ber. 104, 1562-1572 (1971).

332. R. HUISGEN - Avances en las reacciones electrocíclicas. Revista de la Real Academia de Ciencias Exactas, fisicas y Naturales de Madrid, LXV, 267-292 (1971). 
333. R. HUISGEN - Nuevas aportaciones a las cicloadiciones 1,3-dipolares. Revista de la Real Academia de Ciencias Exactas, Fisicas Naturales de Madrid, LXV, 293-319 (1971).

334. G. SCHRÖDER, G. KIRSCH, J.F.M. OTH, R.HUISGEN, W.E. KONZ, U. SCHNEGG - Zur Darstellung und Valenztautomerie des Fluor-cyclooctatetraens. Chem. Ber. 104, 2405-2411 (1971).

335. J. GASTEIGER, G.E. GREAM, R. HUISGEN, W.E. KONZ, U. SCHNEGG - Chlor-, Brom- und Jod-cyclooctatetraen: Darstellung und einige Reaktionen. Chem.Ber. 104, 2412-2419 (1971).

336. J.H. HALL, R. HUISGEN - Cycloadditions of 1,2,3-Triphenylaziridine via the Azomethine Ylide. J.Chem.Soc., Chem.Commun., 1187-1188 (1971).

337. J.H. HALL, R. HUISGEN, C.H. ROSS, W. SCHEER - Evidence for cis- and trans1,2,3-Triphenylaziridine from the Ring-opened Azomethine Ylides. J.Chem. Soc., Chem.Commun., 1188-1190 (1971).

338. H. HAMBERGER, R. HUISGEN - Cycloadditions of a Carbonyl Ylide from 2,3-Dicyano2,3-diphenyloxiran. J.Chem. Soc., Chem.Commun., 1190-1192 (1971).

339. A. DAHMEN, H. HAMBERGER, R. HUISGEN, Y.MARKOWSKI - Conrotatory Ring Opening of Cyanostilbene Oxides to Carbonyl Ylides. J.Chem. Soc., Chem. Commun., 11921194 (1971).

340. E. FUNKE, R. HUISGEN - Ketenoide Reaktionsweise eines mesoionischen Oxazolons(5). Chem. Ber. 104, 3222-3228 (1971).

341. R. HUISGEN - Ring Opening Reactions of Aziridines and Oxiranes. XXIIIrd Intemational Congress of Pure and Applied Chemistry, S. 175-195. Butterworths London, 1971.

342. P. EBERHARD, R. HUISGEN - Steric Course and Regioselectivity in the Cycloodditions of Diczoacetic Ester to trans- and cis-Cinnamic Ester. Tetrahedron Lett. 4337-4342 (1971).

343. R. HUISGEN, P. EBERHARD - 1,3-Dipolar Cycloadditions of Aliphatic Diazo Compounds to $\alpha, \beta$-Unsaturated Carboxylic Esters - Spatial and Electronic Effects on the Steric Course. Tetrahedron Lett. 4343-4346 (1971). 
344. R. HUISGEN - Costin D. Nenitzescu, 15.7.1902-28.7.1970 (Nachruf). Bayer. Akodemie der Wissenschaften, Jahrbuch 1971, S. 221-227, Munchen.

345. P. EBERHARD, R. HUISGEN - Formation of an Allyl Anion from 1-Ryrazolin-4-yl Anion by Cycloreversion. Electronic Prototype of 1,3-Dipolar Cycloaddition. J.Am. Chem. Soc. 95, 1345-1346 (1972).

346. R. HUISGEN, P. EBERHARD - Electrocyclic Ring Opening of a Cyclopropyl Anion to an Ally! Anion. J.Am. Chem. Soc. 95, 1346-1348 (1972).

347. R. HUISGEN, H. STANGL, H. STURM, R. RAAB, K. BUNGE - 1,3-Dipolare Cycloodditionen, 62. Benzonitril-(4-nitro-benzylid) und seine Reaktionen mit CCDoppel- und CC-Dreifachbindungen. Chem. Ber. 105, 1258-1278 (1972).

348. K. BUNGE, R. HUISGEN, R. RAAB, H. STANGL - 1,3-Dipolare Cycloadditionen, 63. Die Anlagerung aromatischer Nitril-ylide an Carbonylverbindungen. Chem. Ber. $105,1279-1295$ (1972).

349. K. BUNGE, R. HUISGEN, R. RAAB - Eine Tautomerie bei Imidsdure-chloriden. Chem. Ber. 105, 1296-1306 (1972).

350. K. BUNGE, R. HUIS GEN, R. RAAB, H. J. STURM - 1,3-Dipolare Cycloadditionen, 64. Weitere Umsetzungen von Nitril-yliden mit Hetero-Mehrfachbindungen. Chem. Ber. 105, 1307-1323 (1972).

351. R. HUISGEN, R. SUSTMANN, K. BUNGE - 1,3-Dipolare Cycloadditionen, 65. Zur Frage der primuren 1, 1-Addition bei den Cycloadditionen der Nitrilium- und Diazonium-betaine. Chem. Ber. 105, 1324-1339 (1972).

352. R. HUISGEN, W.E. KONZ, U. SCHNEGG - 1,4-Cycloadditionen des N-Phenylazodicarbonsdure-imids an Cyclooctatetraen und dessen Halogen-Derivate. Angew. Chem. 84, 765-766 (1972); Angew. Chem., Int. Ed. Engl. 11, 715-716 (1972).

353. J. GASTEIGER, R. HUISGEN - 1,4-Cycloadditionen des Tetracyandthylens an Methoxy- und Phenoxycyclooctatetroen. Angew. Chem. 84, 766 (1972); Angew. Chem., Int. Ed. Engl. 11, 716 (1972). 
354. R. HUISGEN, W. MACK - 1,3-Dipolare Cycloadditionen, 66. 1,3,4-Dioxazole aus Nitriloxiden und Carbonylverbindungen. Chem. Ber. 105, 2805-2814 (1972).

355. R. HUISGEN, W. MACK - 1,3-Dipolare Cycloadditionen, 67. Reaktionen der Nitriloxide mit Thiocarbonylverbindungen. Chem. Ber. 105, 2815-2824 (1972).

356. K. BAST, M. CHRISTL, R. HUISGEN, W. MACK - 1,3-Dipol are Cycloadditionen, 68. Additionen der Nitriloxide an CN-Mehrfachbindungen. Chem. Ber. 105, 2825-2840 (1972).

357. H. BLASCHKE, E. BRUNN, R. HUISGEN, W. MACK - 1,3-Dipolare Cycloadditionen, 69. Reaktionen aromatischer Nitriloxide mit Azodicarbonester und verwandten Verbindungen. Chem.Ber. 105, $2841-2853$ (1972).

358. J. GASTEIGER, R. HUISGEN - 9-Thiobicyclo(4.2.1)nono-2,4,7-triene 9,9-Dioxide. J.Am. Chem. Soc. $94,6541-6543$ (1972).

359. R. HUISGEN, J. GASTEIGER - cis,trans Isomerization of 8-Chlorohomotropylium Salts and of 7,8-Dichloro-cycloocta-1,3,5-triene. Tetrahedron Lett. 3661-3664 (1972).

360. J. GASTEIGER, R. HUISGEN - The Formation of 8-Substituted Homotropylium Salts from Cycloocta-1,3,5-triene Derivatives. Tetrahedron Lett. 3665-3670 (1972).

361. R. HUISGEN, J. GASTEIGER - Der Primärschritt der Halogenierung des Cyclooctatetraens. Angow. Chem. 84, 1187-1188 (1972); Angew. Chem., Int. Ed. Engl. 11, $1104-1105$ (1972).

362. R. HUISGEN, G. STEINER - Nonstereospecificity in the (2+2) Cycloadditions of Tetracyanoethylene to Enol Ethers. J.Am. Chem. Soc. 95, 5054-5055 (1973).

363. R. HUISGEN, G. STEINER - Reversibility of Zwitterion Formation in the $(2+2)$ Cycloaddition of Tetracyanoethylene to Enol Ethers. J.Am. Chem. Soc. 95, 50555056 (1973).

364. G. STEINER, R. HUISGEN - Tetracyanoethylene and Enol Ethers. Dependence of Cycloaddition Rate on Solvent Polarity. J.Am. Chem. Soc. 95, 5056-5058 (1973). 
365. R. HUISGEN, G. STEINER - Tetracyanoethylene and Enol Ethers: Rates of 2+2 $\rightarrow 4$ Cycloadditions and Structural Variation of the Enol Ether. Tetrahedron Lett. 3763-3768 (1973).

366. G. STEINER, R. HUISGEN $-2+2 \rightarrow 4$ Cycloadditions of Tetracyanoethylene to Enol Ethers; Activation Parameters as Mechanistic Criteria. Tetrahedron Lett. 3769-3772 (1973).

367. K. BAST, M. CHRISTL, R. HUISGEN, W. MACK, R. SUSTMANN - 1,3-Dipolare Cycloadditionen, 70. Additionen des Benzonitriloxids on olefinische und acetylenische Dipolarophile. Chem. Ber. 104, 3258-3274 (1973).

368. M: CHRISTL, R. HUISGEN, R. SUSTMANN - 1,3-Dipolare Cycloadditionen, 71. Zur Anlagerung des Benzonitriloxids an $\alpha, \beta$-ungesdttigte Carbonsdureester. Chem. Ber. $106,3275-3290$ (1973).

369. R. HUIS GEN, M. CHRISTL - 1,3-Dipolare Cycloadditionen, 72. Reaktionen der Knallsaure mit ungesättigten Verbindungen. Chem. Ber. 106, $3291-3311$ (1973).

370. K. BAST, M. CHRISTL, R. HUIS GEN, W. MACK - 1,3-Dipolare Cycloadditionen, 73. Relative Dipolarophilen-Aktivitaten bei Cycloadditionen des Benzonitriloxids. Chem. Ber. 106, 3312-3344 (1973).

371. M. CHRISTL, R. HUISGEN - 1,3-Dipolare Cycloadditionen, 74. Orientierungsphänomene bei Cycloadditionen aliphatischer und aromatischer Nitriloxide on $\alpha, \beta$-ungesdttigte Carbonester. Chem.Ber. 106, 3345-3367 (1973).

372. R. HUISGEN, R. SCHUG, G. STEINER - Abfangen des 1,4-Dipols bei der 2+2Cycloaddition des Tetracyandthylens an Enoluther. Angew. Chem. 86, 47-48 (1974); Angew. Chem., Int. Ed. Engl. 13, 80-81 (1974).

373. R. HUISGEN, R. SCHUG, G. STEINER - Zur Konformation der zwitterionischen Zwischenstufen ous Tetracyonathylen und Alkyl-propenylathem. Angew. Chem. 86, 48-49 (1974); Angew. Chem., Int. Ed. Engl. 13, $81-82$ (1974).

374. W. FLIEGE, R. HUISGEN - 1,3-Dipolare Cycloadditionen, 75. Zur exo-Anlagerung des Dipheny Initrilimins und Benzonitriloxids an Norbornen und Apobornen. Liebigs Ann. Chem. 1973, 2038-2047. 
375. L.A. FEILER, R. HUISGEN, P. KOPPITZ - 2:I Adducts of Diphenylketene and Norbornene. J.Am. Chem. Soc. 96, 2270-2271 (1974).

376. L.A. FEILER, R. HUISGEN, P. KOPPITZ - Carboxylic Anhydrides and Diphenylketen, a New Reaction Mode of Ketens. J.Chem. Soc., Chem. Comm., 405-406 (1974).

377. P. CARAMELLA, R. HUIS GEN, B. SCHMOLKE - Substituent Effects in the Ring Enlargement of $\mathrm{N}$-Arylidene-2,2-diphenylcyclopropylamines. J.Am. Chem. Soc. 96, 2997-2999 (1974).

378. P. CARAMELLA, R. HUISGEN, B. SCHMOLKE - Racemization and Ring Expansion of N-Arylidene-2,2-diphenylcyclopropylamines via a Common Trimethylene Intermediate. J.Am. Chem. Soc. 96, 2999-3000 (1974).

379. R. HUISGEN, P. KOPPITZ - 2:1-Addukte des Diphenylketens mit a-Methylstyrol und 1,1-Diphenyldthylen. Chem. -Ztg. 98, 461-462 (1974).

380. R. HUISGEN, R. SCHUG - Concentration Dependence of the Rate Constants for 2+2 Cycloodditions of Tetracyanoethylene. J.Chem.Soc., Chem.Comm., 59-60 (1975).

381. R. SCHUG, R. HUISGEN - 1,4-Dipolar Cycloadditions as Trapping Reactions for Zwitterionic Intermediates of $2+2$ Cycloadditions. J.Chem. Soc., Chem. Comm., 60-61 (1975).

382. C. -C. TAN, C. RÜCHARDT, G. BINSCH, D. HÖFNER, R. HUISGEN, and H. NAKATEN - Aromatische Diazoniumsalze, VI. Nitrosoacyl-amine und Diazoester, XIV: Eine neve Reaktionsweise des N-Nitrosoacetanilids. Chem.Ber. 108, $1027-1035$ (1975).

383. H. MAYR, R. HUISGEN - Structural Assignments of Stereoisomeric Cyclobutanones by Substituent Increments on Chemical Shifts. Tetrahedron Lett. 1349-1352 (1975).

384. H. MAYR, R. HUISGEN - Eine neve Synthese von Cyclobutenonen. Angew. Chem. 87, 491 (1975); Angew. Chem., Int. Ed. Engl. 14, 499-500 (1975). 
385. R. HUISGEN, H. MAYR - Kinetics of 2+2 Cycloadditions of Diphenylketene to Enol Ethers; the Structure of the Orientation Complexes. Tetrahedron Lett. 29652968 (1975).

386. R. HUISGEN, H. MAYR - Steric Course, Kinetics and Mechanism of the 2+2 Cycloadditions of Alkylphenylketenes to Ethyl cis- and trans-Propenyl Ether. Tetrahedron Lett. 2969-2972 (1975).

387. I. KARLE, J. FLIPPEN, R. HUISGEN, R. SCHUG - $(2+2)$ Cycloadditions of Tetracyanoethylene to Enol Ethers. Structure of the Product of Interception with Alcohol. J. Am. Chem. Soc. 97, 5285-5287 (1975).

388. A. MAQUestiau, Y. VAN HAVERBEKe, R. FLAMMANG, R. HUISGEN, Y.B. CHAE - Fragmentation d'Hétérocycles sous I'Impact Electronique. Détermination de schémas de frogmentation par la technique C.I.D./M.I.K.E. Bull.Soc.Chim. Belg. 84, 1179-1187 (1975).

389. R. HUISGEN, A. OHTA, J. GEITTNER - 1,3-Dipolar Cycloadditions, 76. Cycloadditions of Diazomethane to Substituted Butadienes. Chem. Pharm. Bull. Japan, 23, 2735-2743 (1975).

390. R. HUIS GEN, H. MAYR - Reactions of Cyclobutenones with Nucleophilic Reagents via Vinylketen Intermediates. J.Chem. Soc., Chem. Commun., 55-56 (1976).

391. H. MAYR, R. HUISGEN - Kinetics and Mechonism of the Conversion of Cyclobutenones to Vinylketens. J.Chem. Soc., Chem. Commun., 57-58 (1976).

392. R. HUISGEN - The Concerted Nature of 1,3-Dipolar Cycloadditions and the Question of Diradical intermediates. J. Org. Chem. 41, 403-419 (1976).

393. V. MARKOWSKI, R. HUISGEN - Disrotatory Photoconversion of cis, trans Isomeric Oxiranes to Cabonyl Ylides. Tetrahedron Lett. 4643-4646 (1976).

394. R. HUISGEN - Eiji Ochiai, 26.6.1898 - 4.11.1974 (Nachruf). Bayer. Akademie der Wissenschaften, Jahruch 1976, S. 215-217, Munchen.

395. R. HUISGEN - Rudolf Criegee, 23.5.1902 - 7.11.1975 (Nachruf). Bayer. Akademie der Wissenschaften, Jahrbuch 1976, S. 235-238, MUnchen. 
396. R. HUIS GEN, R. SCHUG - New Mechanistic Criterion for Early and Late Transition States. J.Am. Chem. Soc. 98, 7819-7821 (1976).

397. R. HUISGEN, S. SUSTMANN - 1,3-Dipolar Cycloadditions of Carbonyl Ylides to Thiocarbonyl and Carbonyl Compounds. Heterocycles (Sendai) $\underline{5}, 141-146$ (1976).

398. H. HAMBERGER, R. HUISGEN, V. MARKOWSKI, S. SUSTMANN - The Reactions of Carbonyl Ylides with Azodicarboxylic Esters. Heterocycles (Sendai) 5, 147-151 (1976).

399. R. HUISGEN, R. SCHUG, G. STEINER - Cycloadditions $2+2$ par des intermédiaires dipolaires-1,4. Bull. Soc. Chim. France, 1813-1820 (1976).

400. R. HUISGEN - 2+2 Cycloadditionen über polare Zwischenstufen. Chimia 31, 13-14 (1977).

401. R. HUISGEN, R. FLEISCHMANN, A. ECKELL - Die Reaktion aromatischer Diazocyanide mit Diazcalkanen; eine Klasse stabiler kristalliner Azomethin-imine. Chem. Ber. 110, 500-513 (1977).

402. R. HUISGEN, R. FLEISCHMANN, A. ECKELL - Umsetzungen von $C, C, N^{\alpha}-T$ riaryl$N^{\beta}$-cyan-azomethiniminen mit Sduren, Basen und Reduktionsmitteln. Chem. Ber. 110, $514-521$ (1977).

403. R. HUISGEN, A. ECKELL - 1,3-Dipolare Cycloadditionen, 78. Additionen des $C-\left(2,2^{\prime}-B i p h e n y l y l e n\right)-N^{\alpha}-(4-c h l o r p h e n y l)-N^{\beta}$-cyan-azomethinimins an Styrol, Alkene, Enolether und Enamine. Chem. Ber. 110, 522-539 (1977).

404. R. HUISGEN, A. ECKELL - 1,3-Dipolare Cycloadditionen, 79. Umsetzungen des $C-\left(2,2^{\prime}\right.$-Biphenylylen) $-N^{\alpha}$-(4-chlorpheny 1$)-N^{\beta}$-cyan-azomethinimins mit $\alpha, \beta$-ungesättigten Carbonsaureestern und Nitrilen. Chem. Ber. 110, 540-558 (1977).

405. A. ECKELL, R. HUISGEN - 1,3-Dipolare Cycloadditionen, 80. C,C,N ${ }^{\alpha}$-Triaryl$N^{\beta}$-cyan-azomethinimine und acetylenische Dipolarophile. Chem. Ber. 110, 559-570 (1977).

406. A. ECKELL, R. HUISGEN - 1,3-Dipolare Cycloadditionen, 81. Anlagerungen von $C, C, N^{\alpha}$-Triaryl- $N^{\beta}$-cyon-azomethiniminen an $C N-$ Mehrfachbindungen. Chem. Ber. $110,571-577$ (1977). 
407. A. ECKELL, M.V. GEORGE, R. HUISGEN, A.S. KENDE - 1,3-Dipolare Cycloadditionen, 82. Kinetik und Mechan ismus der Cycloodditionen des $C-\left(2,2^{\prime}-8 i p h e n-\right.$ ylen)- $N^{\alpha}$-(4-chlorphenyl)-N $N^{\beta}$-cyan-azomethinimins. Chem. Ber. 110, 578-595 (1977).

408. J. GEITTNER, R. HUISGEN, R. SUSTMANN - Kinetics of 1,3-Dipolar Cycloaddition Reactions of Diazomethane; A Correlation with HOMO-LUMO Energies. Tetrahedron Lett. , $881-884$ (1977).

409. R. SUSTMANN, E. WENNING, R. HUISGEN - Perturbational Analysis of 1,3-Dipolar Cycloaddition Reactions of Diazomethane. Tetrahedron Lett., 877-880 (1977).

410. R. KRISCHKE, R. GRASHEY, R. HUISGEN - 1,3-Dipolare Cycloadditionen, 83. Pyridinium-N-imid als additionsbereites Azomethinimin. Liebigs Ann. Chem. 1977, 498-505.

411. R. HUISGEN, R. GRASHEY, R. KRISCHKE - 1,3-Dipolare Cycloadditionen, 84. Additionen mit Chinolinium-, lsochinolinium- und Phenanthridinium-N-imid. Liebigs Ann. Chem. 1977, 506-527.

412. R. HUISGEN - Tetracyanoethylene and Enol Ethers. A Model for $2+2$ Cycloadditions via Zwitterionic Intermediates. Acc.Chem. Res. 10, 117-124 (1977).

413. R. HUISGEN - Can Tetramethylene Intermediates Be Intercepted ? Acc. Chem. Res. 10, 199-206 (1977).

414. V. MARKOWSKI, R. HUISGEN - Kinetics of the Electrocyclic Ring Opening of a-Cyano-cis- and trans-stilbene Oxides. J.Chem.Soc., Chem.Commun., 439440 (1977).

415. R. HUISGEN, V. MARKOWSKI - Stereochemistry of the Thermal Electrocyclic Ring Opening of a-Cyano-cis-stilbene Oxide. J.Chem. Soc., Chem. Commun., 440-442 (1977).

416. R. HUISGEN, V. MARKOWSKI, H. HERMANN - Flash Photolysis of a-Cyano-cisand -trans-Stilbene Oxide; Energy Profile of $c$ is,trans Isomerization via Carbonyl Ylides. Heterocycles (Sendai) 7 , 61-66 (1977). 
417. R. HUISGEN - Elektrocyclische Ringöffnungen von Ethylenoxiden. Angew. Chem. 89, 589-602 (1977); Angew. Chem., int. Ed.Engl. 16, 572-585 (1977).

418. R. HUISGEN, Th. SCHMIDT - 1,3-Dipolare Cycloadditionen, 85. Zur Reaktion mesoionischer Oxazolone mit Schwefelkohlenstoff. Liebigs Ann. Chem. 1978, 29-40.

419. R. HUISGEN - Das Portrait: Rudolf Criegee (1902 - 1975). Chemie in unserer Zeit 2, 49-55 (1978); Engl.Übers.: J.Chem. Educ. 56, 369-374 (1979).

420. R. HUISGEN, J. PLUMET ORTEGA - Evidence for a Zwitterionic Intermediate in the 2+2 Cycloaddition of Tetracyanoethylene to a trans-fixed 1,3-Diene. Tetrahedron Lett. 3975-3978 (1978).

421. W. BIHLMAIER, J. GEITTNER, R. HUIS GEN, H.-U. REISSIG - The Stereospecificity of Diazomethane Cycloadditions. Heterocycles (Sendai) 10, 147-152 (1978).

422. L. FIŠERA, J. GEITTNER, R. HUISGEN, H.-U. REISSIG - Cycloaddition Rates of Diazomethane and Diphenyldiazomethane. Heterocycles (Sendai) 10, 153-158 (1978).

423. R. HUISGEN, J. GEITTNER - Kinetics of 1,3-Dipolar Cycloaddition Reactions of Aryl-and Diaryldiazomethanes. Heterocycles (Sendai) 11, 105-108 (1978).

424. J. GEITTNER, R. HUISGEN, H.-U. REISSIG - Solvent Dependence of Cycloaddition Rates of Phenyldiazomethane and Activation Parameters. Heterocycles (Sendai) $11,109-112$ (1978).

425. J.v. JOUANNE, H. KELM, R. HUISGEN - Effect of Pressure on the $(2+2 \rightarrow 4)$ Cycloaddition Reaction of Tetracyanoethylene to Enol Ethers. J.Am. Chem. Soc. 101, 151-158 (1979).

426. M. MINGIN, R. HUISGEN - The Intermolecular Self-Condensation of 2,4-Pentanedione under the Influence of Potassium Fluoride in Dimethylformamide. Tetrahedron Lett. 719-720 (1979).

427. R. HUISGEN, H.-U. REISSIG - Cycloadditionen von a-Diazocarbonylverbindungen an Enamine. Angew. Chem. 91, 346-347 (1979); Angew. Chem., Int. Ed. Engl. $18,330-331$ (1979). 
428. R. HUISGEN, W. BIHLMAIER, H.-U. REISSIG - Azokupplung von a-Diazocarbonyl-

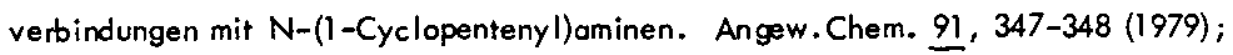
Angew. Chem., Int. Ed. Engl. 18, 331-332 (1979).

429. R. BERMES, A. DAHMEN, H. HAMBERGER, R. HUISGEN, P. de MARCH, V. MARKOWSKI - Synthesis of Hydrofurans by Cycloadditions of Carbonyl Ylides. Third International Symposium of Furan Chemistry, Collection of Lectures, The Czechoslovak Chemical Society, Bratislava, 1979, S. 20-38.

430. W. BIHLMAIER, R. HUISGEN, H.-U. REISSIG, S. VOSS - Reactivity Sequences of Dipolarophiles Towards Diazocarbonyl Compounds - MO Perturbation Treatment. Tetrahedron Lett. $2621-2624$ (1979).

431. R. HUISGEN, H. -U. REISSIG, H. HUBER - Diazocarbonyl Compounds and I-Diethylaminopropyne. J.Am. Chem. Soc. 101, 3647-3648 (1979).

432. H. -U. REISSIG, R. HUISGEN - Conducted Tour Mechanism of Ester Group Migration in a $3 \mathrm{H}$-Pyrazole. J. Am. Chem. Soc. 101,3648 (1979).

433. R. HUISGEN, H. -U. REISSIG, H. HUBER, S. VOSS - a-Diazocarbonyl Compounds and Enamines - A Dichotomy of Reoction Paths. Tetrahedron Lett. 2987-2990 (1979).

434. H. GRAF, R. HUISGEN - Rates and Mechanism of $(2+2)$ Cyclooddition Reactions of Tetracyanoethylene to Thioenol Ethers. J. Org. Chem. 44, 2594-2595 (1979).

435. R. HUISGEN, H. GRAF - Stereochemistry of Tetracyanoethylene Cycloadditions to Thioenol Ethers. J.Org. Chem. 44, 2595-2596 (1979).

436. R. HUIS GEN, H. -U. REISSIG - Cycloadditions to Methyl 3,3-Dimethyl-3H-pyrazole-5-carboxylate. J.Chem. Soc., Chem. Commun. 568-570 (1979).

437. R. HUISGEN, J. KOSZINOWSKI, A. OHTA, R. SCHIFFER - Cycloadditionen der Diazoalkene an 1-Alkene. Angew. Chem. 92, 198-199 (1980); Angew. Chem., Int. Ed. Engl. 19, 202-203 (1980). 
438. R. HUISGEN, P.H. J. OOMS, M. MINGIN, N.L. ALLINGER - Exceptional Reactivity of the Bicyclo(2.2.1)heptene Double Bond. J. Am. Chem. Soc. 102, $3951-3953(1980)$.

439. R. HUISGEN - Cycloaddition Mechanism and the Solvent Dependence of Rate. Pure Appl.Chem. 52, 2283-2302 (1980).

440. J.L. FLIPPEN-ANDERSON, I. KARLE, R. HUISGEN, H.U. REISSIG - Trimerization of an Azomethine Imine by Three Successive 1,3-Dipolar Cyclooddition Reactions. Angew. Chem. 92, 936-937 (1980); Angew. Chem., Int. Ed. Engl. 19, 906-907 (1980).

441. R. HUISGEN - 1,5-Elektrocyclisierungen - ein wichtiges Prinzip der Heter $\alpha y c l e n-$ Chemie. Angew. Chem. 92, 979-1005 (1980); Angew. Chem., Int. Ed. Engl. 19, 947-973 (1980).

442. R. HUISGEN - Recent Contributions to the Mechanism of Concerted and Non-Concerted Cycloadditions. Pure and Appl. Chem. 53, 171-187 (1981).

443. R. HUISGEN, K. MATSUMOIO, C.H. ROSS - Cycloodditions of 1-Substituted cis- and trans-2,3-Diphenylaziridines $v$ ia Azomethine Ylides. Heterocycles (Sendai) $15,1131-1136$ (1981).

444. R. HUISGEN - An Important Prinziple of the Chemistry of Heterocycles: Electrocyclic Reactions of the Pentadienyl Anion Type. Khimiya Geterotsiklicheskikh Soedenenii (Kazan, UdSSR), 579-598 (1981) (orig. russisch).

445. R. HUISGEN, M. P. BOSCH VERDEROL, A. GIEREN, V. LAMM - Reaktion von Dibenzoyldiazomethan mit 1-Diethylaminopropin. Angew. Chem. 93, 710-712 (1981); Angew. Chem., Int. Ed. Engl. 20, $694-695$ (1981).

446. I. KALWINSCH, R. HUISGEN - The Reaction of Diazoocetic Ester with Thiobenzophenone. Tetrahedron Lett. 3941-3944 (1981).

447. R. HUISGEN - Neves uber Azomethin-imine - Abentever ei nes heterocyclischen Systems. Chimia 35, 344-346 (1981). 
448. I. KALWINSCH, LI XINGYA, J. GOTISTEIN, R. HUISGEN - Diazomerhane and Thiobenzophenone; Mechanistic Elucidation of the Schonberg Reaction. J.Am. Chem. Soc. 103, 7032-7033 (1981).

449. R. HUISGEN, F. PALACIOS GAMBRA - 1,3-Dipolar Cycloodditions of Aromatic Azoxy Compounds to Strained Cycloalkenes. Tetrahedron Lett. 55-58 (1982).

450. R. HUISGEN, F. PALACIOS GAMBRA - 1,3-Dipolare Cycloadditionen, 86: Aromatische Azoxyverbindungen und gespannte Cycloalkene. Chem. Ber. 115, 2242-2255 (1982).

451. P. de MARCH, R. HUISGEN - Carbonyl Ylides from Aldehydes and Carbenes. J.Am. Chem. Soc. 104, 4952 (1982).

452. R. HUISGEN, P. de MARCH - Three-Component Reactions of Diazomalonic Ester, Benzaldehyde, and Electrophilic Olefins. J.Am. Chem. Soc. 104, 4953-4954 (1982).

453. R. HUISGEN - Neves Uber Thiocarbonyi-ylide. Festschrift anlaßlich des 60. Geburtstags von Prof. Dr. K. Weissermel, S. 21-23, Hoechsł AG, Frankfurt 1982.

454. R. HUISGEN - Reaktionsmechanismen und Prdparative Otganische Chemie. Forschung in der Bundesrepublik Deutschland; Beispiele, Kritik, Vorschläge (im Auftrag der Deutschen Forschungsgemeinschaft), S. $621-633$, Verlag Chemie, Weinheim, 1983.

455. R. HUISGEN - Fritz Krobnke - Forscher und Mensch 1903-1981. Chem. Ber. 116, XIV $-X X(1983)$.

456. G. SWIETON, J.v. JOUANNE, H. KELM, R. HUISGEN - The Effect of Pressure on the $(2+2 \rightarrow 4)$ Cycloaddition of Diphenylketen to Enol Ethers. J. Chem. Soc. Perkin Trans. II, 37-43 (1983).

457. G. SWIETON, J.v. JOUANNE, H. KELM, R. HUISGEN - Effect of Pressure on the 1,3-Dipolar Cycloaddition Reoction of Diphenyldiazomethane to CarbonCarbon Multiple Bonds. J. Org. Chem. 48, 1035-1040 (1983). 
458. R. HUISGEN, W. FLIEGE, W. KOLBECK - 1,3-Dipolare Cycloadditionen, 87 : Zur Kenntnis des N-Phenylnitrilimins. Chem. Ber. 116, 3027-3038 (1983).

459. W. FLIEGE, R. HUISGEN, J.S. CLOVIS, H. KNUPFER - 1,3-Dipolare Cycloadditionen, 88 : C-Methyl-N-phenylnitrilimin und die Regiochemie seiner Cycloadditionen. Chem. Ber. 116, 3039-3061 (1983).

460. J.S. CLOVIS, W. FLIEGE, R. HUISGEN - 1,3-Dipolare Cycloadditionen, 89 : Neue Beitröge zur Chemie des Diphenylnitrilimins. Chem. Ber. 116, 3062-3070 (1982).

461. R. HUISGEN - Neve Cycloadditionen von Azomethiniminen. Wiss. Z. KariMarx-Univ. Leipzig, Math. -Naturwiss.R., 32, 395-406 (1983).

462. Li XINGYA, R. HUISGEN - New Reactions of Thiobenzophenone S-Methylide. Tetrahedron Lett. 4181-4184 (1983).

463. R. HUISGEN, LI XINGYA - The Activity Scale of Dipolarophiles versus Thiobenzophenone S-Methylide. Tetrahedron Lett. 4185-4188 (1983).

464. W. FLIEGE, R. HUISGEN, W. KOLBECK, V. WEBERNDÖRFER - 1,3-Dipolore Cycloadditionen, 90 : Diphenylnitrilimin und substituierte Butadiene; Substituenteneffekte und Geschwindigkeit der Cycloaddition. Chem. Ber. 116, $3438-3460$ (1983).

465. R. HUISGEN, LI XINGYA - New Thiocarbony! Ylides from Thiobenzophenone. Heterocycles (Sendai) 20, 2363-2368 (1983).

466. R. HUISGEN, K. NIKLAS - The Chemistry of an Isolable Azometh in Ylide. Heterocycles (Sendai) 22, 21-26 (1984).

467. F. BRONBERGER, R. HUISGEN - Diels-Alder Reactions of 1,3-Dienes with 4-Nitrobenzenediazonium Salt as a Dienophile. Tetrahedron Lett. 57-60 (1984).

468. R. HUISGEN, F. BRONBERGER - 4-Nitrobenzenediazonium Salt and Cyclopentadiene. Tetrahedron Lett. $61-64$ (1984). 
469. F. BRONBERGER, R. HUISGEN - The Arenediazonium Ion as a Dipolarophile. Tetrahedron Lett. $65-68$ (1984).

470. W. FLIEGE, R. GRASHEY, R. HUISGEN - 1,3-Dipolare Cycloadditionen, 91 : Die Chemie des N-Methyl-C-phenyinitrilimins. Chem. Ber. 117, 1194-1214 (1984).

471. R. HUISGEN - 1,3-Dipolar Cycloodditions - Introduction, Survey, Mechanism. in "1,3-Dipolar Cyclooddition Chemistry", Herausgeber A. Padwa, S. 2-176, John Wiley and Sons, New York 1984.

472. R. HUISGEN, C. FULKA, I. KALWINSCH, LIXINGYA, G. MLOSTON, J.R. MORAN, A. PRÖBSTL - Recent Developments of the Chemistry of Thiocarbonyl Ylides. Bull. Soc. Chim. Belg. 93, $511-532$ (1984).

473. R. HUISGEN - Karl Freudenberg, 29.1.1886 - 3.4.1983 (Nachruf). Bayer. Akademie der Wissenschaften, Jahrbuch 1984, S. 246-249.

474. R. HUISGEN, G. MLOSTON - Adamantanethione and Diazomethane; A Re-Examination. Telrahedron Lett. 1049-1052 (1985).

475. G. MLOSTON, R. HUISGEN - Acid-Base Reactions of 1,3,4-Thiadiazolines and Thiocarbony! Ylides; 1,3,4-Thiadiazoline-2-spiro-2'-adamantane. Terrahedron Lett. 1053-1056 (1985).

476. R. HUISGEN, J.R. MORAN - Tropothione and Diazomethane; Revision and New Approch. Tetrahedron Lett. $1057-1060$ (1985).

477. J.R. MORAN, R. HUISGEN, I. KALWINSCH - Thiobenzophenone and Tetracyanoethylene. Tetrahedron Lett. 1849-1852 (1985).

478. I.L. KARLE, J.L. FLIPPEN-ANDERSON, and R. HUISGEN-Cycloaddition of Isoquinolinium-N-phenylimide with Dimethyl Fumarate and Dimethyl Maleate and the ir Acid Rearrangement Products: $\mathrm{C}_{21} \mathrm{H}_{20} \mathrm{~N}_{2} \mathrm{O}_{4}$ (6) and $\mathrm{C}_{24} \mathrm{H}_{22} \mathrm{~N}_{2} \mathrm{O}_{6}$ (7). Acta Cryst. C 41, 1095-1100 (1985). 
479. R. HUISGEN, G. MLOSTON, and A. PRÖBSTL - The Chemistry of 1,3,4-Thiadiazoline-2-spiro-2' -fenchane. Tetrahedron Lett. $4431-4434$ (1985).

480. G. MLOSTON and R. HUISGEN - Schönberg Reactions of Adamantanethione S-Methylide. Heterocycles (Sendai) 23, 2201-2206 (1985).

481. R. HUISGEN, G. MLOSTON, and C. FULKA - 1,3-Cycloodditions of 2,2,4,4-Tetramethylcyclobutane-l -one-3-thione S-Methylide. Heterocycles (Sendai) 23, 2207-2212 (1985).

482. R. HUISGEN and R. WEINBERGER - Are Any Non-stereospecific 1,3-Dipolar Cycloadditions known? A Revision. Tetrahedron Lett. 5119-5122 (1985).

483. R. HUISGEN - Adolf von Baeyers wissenschaftliches Werk - Ein Vermächtnis. Angew. Chem. 98, 297-311 (1986); Angew. Chem., Int. Ed.Engl. 25, 297-311 (1986).

484. R. HUISGEN, A. MITRA, and J.R. MORAN - The Astounding Reaction of Diazomethane with Dimethyl 2,3-Dicyanofumarate. Heterocycles 24, 2429-2436 (1986).

485. R. HUISGEN, G. MLOSTON, and E. LANGHALS - First Two-Step 1,3-Dipolar Cycloadditions: Nonstereospecificity. J.Am. Chem. Soc. 108, $6401-6402$ (1986).

486. R. HUISGEN, E. LANGHALS, and H. NÖTH - Further Contributions to the Stereospecificity of 1,3-Dipolar Cycloadditions of Thiocarbonyl Ylides. Tetrahedron Lett. 27, 5475-5478 (1986).

487. R. HUISGEN and XIA PENG - 1,3-Cycloadditions of a Thionitroso S-Sulfide. Tetrahedron Lett. 6063-6066 (1986).

488. R. HUISGEN, G. MLOSTON, and E. LANGHALS - The First TwoSiep 1,3-Cycloadditions: Interception of an Intermediate. J. Org. Chem. 51, 4085-4087 (1986). 
489. R. HUISGEN and J. RAPP - Thiocarbonyl S-Sulfides, a New Class of 1,3-Dipoles. J.Am. Chem. Soc. 109, $902-903$ (1987).

490. U. EICHENAUER, R. HUISGEN, A. MITRA, and J.R. MORAN - The Reaction of Aliphatic Diazo Compounds with Highly Electrophilic Ethylene Derivatives. Heterocycles 25, $129-132$ (1987).

491. R. HUISGEN, U. EICHENAUER, E. LANGHALS, A. MIIRA, und J.R. MORAN - 1,3-Dipolore Cycloadditionen, 92. Reaktionen aliphat ischer Diazoverbindungen mit vierfach Acceptor-substituierten Ethylenen. Chem. Ber. 120, 153-158 (1987).

492. R. HUISGEN, A. MITRA und J.R. MORAN - 1,3-Dipolare Cycloadditionen, 93. Überraschungen bei der Umsetzung des 2,3-Dicyanofumarsäure-dimethylesters mit Diazomethan. Chem. Ber. 120, 159 169 (1987).

493. D. BOECKH, R. HUISGEN, and H. NÖIH - Preparation and Con-

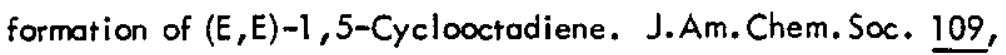
$1248-1249$ (1987).

494. R. HUISGEN, E. LANGHALS, G. MLOSTON, T. OSHIMA, and J. RAPP - New Cycloadditions in Organic Sulfur Chemistry. Lectures in Heterocycl. Chem., Suppl. of J. Heterocycl. Chem. 24 S1 - 511 (1987).

495. R. HUISGEN - Steric Course and Mechainism of 1,3-Dipolar Cycloodditions. Advances in Cycloaddition 1, 1-31 (1988).

496. R. HUISGEN - Georg Wittig 16.6.1897 - 28.8.198 . Jahrbuch der Bayerischen Akademie der Wissenschaften 1987, S. 275 - 278 (1988).

497. R. HUISGEN - From Thiiranes to Thiocarbonyl S-Sulfides; Recent Results. Phosphorus, Sulfur and Silicon, 43, 63-94 (1989). 
498. R. HUISGEN - Kinetics and reaction mechan isms: selected examples from the experience of forty years. Pure Appl. Chem. 61, 613-628 (1989).

499. R. HUISGEN - David Ginsburg 5.9.1920 - 9.3.1988. Jahrbuch der Bayerischen Akademie der Wissenschaften 1988, S. 218 - 221 (1989).

500. R. HUISGEN and E. LANGHALS - Thiones as Superdipolarophiles, letrahedion Lett. 30, 5369 - 5372 (1989).

501. G. MLOSTON, E. LANGHALS, and R. HUISGEN - 1,3-Cycloadditions of Aliphatic Thione S-Methylides to Dimethyl 2,3-Dicyanofumarate and 2,3-Dicyanomaleate; a Test Case for Steric Course and Mechanism. Tetrahedron Lett. 30, 5373 - 5376 (1989).

502. R. HUISGEN, E. LANGHALS, G. MLOSTON, and T. OSHIMA A Stable Seven-membered Ring Ketene Imine From a Thiocarbanyl Ylide and an Acceptor Olefin. Heterocycles 29, 2069 - 2074 (1989).

503. R. HUISGEN, E. LANGHALS, and T. OSHIMA - Ring Contraction of a Cyclic Seven-membered Ketene Imine. Heterocycles 29, 2075 2078 (1989).

504. R. HUISGEN - Theoret ische Weiterbildung der Doktoranden tut not! Nachrichten aus Chemie, Technik und Laboratorium 37, 1257 (1989).

505. R. HUISGEN and G. MLOSTON - Cycloadditions of Dialky/ Thioketone S-Methylides. Tetrahedron Lett. 30, 7041 - 7044 (1989).

506. G. MLOSTON and R. HUISGEN - Open-choin Aliphatic Thiones and Diazomethane; Reactions of 1,3,4-Thiadiazolines and Ihiocarbonyl Ylides. Tetrahedron Lett. 30, 7045 - 7048 (1989).

507. R. HUISGEN and G. MLOSTON - Reactions of a Tetrasubstituted Thiocarbonyl Ylide; New Evidence for Two-step Cycloadd itions. Heterocycles $30,737-740$ ( 1990). 
508. R. HUISGEN, E. LANGHALS, and H. NÖTH - A Cristalline Seven-Membered Cyclic Ketene Imine from a Thiocarbonyl S-Methylide. J. Org. Chem. 55, $1412-1414$ (1990).

509. R. HUISGEN and R. BRÜCKNER - [2+2 ]Cycloadditions of 2,2-Bis(trifluoromethyl)ethylene-1,1-dicorbonitrile with Enol Ethers, 1,1-Dimethylbutadiene, and Allyltrimethylsilane. Tetrahedron Lett. 31, 2553 - 2556 (1990).

510. R. BRÜCKNER and R. HUISGEN - Kinetics of [2+2] Cycloadditions of 2,2-Bis(trifluoromethyl)ethylene-1,1-dicarbonitrile with Enol Ethers, 1, 1-Dime thylbutadiene, and Allyltrimethylsilane. Tetrahedron Lett. 31, $2557-2560$ (1990).

511. R. BRÜCKNER and R. HUISGEN - [2+2]Cycloodditions of 2,2-Bistri fluoromethyl)ethylene-1,1-dicarbonitrile with Vinyl Sulfides and Ketene S, S-Acetals. Tetrahedron Lett. 31, 2561 - 2564 (1990).

512. R. BRÜCKNER, R. HUISGEN, and J.S. SCHMID - 2,2-Bis (trifluoromethyl) ethylene-1, l-dicarbonitrile and Styrenes. A Dichotomy of Cycloaddition Pathways. Tetrahedron Lett. 31, $7129-7132$ (1990).

513. R. BRÜCKNER and R. HUISGEN - 2,2-Bis(trifluoromethyl)ethylene-1,1dicarbonitrile and Styrenes. The Concertedness of the [2+4] Cyclooddition. Tetrohedron Lett. 31, $7133-7136$ (1990).

514. R. BRÜCKNER and R. HUISGEN - The Electron-Deficient Olefin 2,2-Bis(trifluoromethyl)ethylene-1, 1-dicarbonitrile: Hydride Abstractions from Allyl and Isobutenyl Ethers and Thicethers. J. Org.Chem. 56, $1677-1679$ (1991).

515. R. HUISGEN and R. BRÜCKNER - 2,2-Bis (trifluoromethyl)ethylene-1, 1-dicarbonitrile as a Unique Enophile. J. Org. Chem. 56, 1679 - 1681 (1991).

516. R. BRÜCKNER and R. HUISGEN - Electrophilic Substitutions by 2,2-Bis(trifluoromethyl)ethylene-1,1-dicarbonitrile via Addition. Tetrahedron Lett. $\underline{32}$, 1871 - 1874 (1991). 
517. R. BRÜCKNER and R. HUISGEN - Substitutions and Dehydrogenations by 2,2-Bis(trifluoromethyl)ethylene-1,1-dicarbonitrile via Hydride Abstraction. Tetrahedron Lett. 32, 1875 - 1878 (1991).

518. R. HUISGEN, J. PENELLE, G. MLOSTON, A.B. PADIAS, and H.K. HALL, jr.- Can Polymerization Trap Intermediates in 1,3-Dipolar Cycloadditions ? J.Am.Chem.Soc. 114, 266 - 274 (1992).

519. R. SUSTMANN and W. SICKING, R. HUISGEN - Regiochemistry in Cycloadditions of Diazomethane to Thioformaldehyde and Thioketones. J.Org.Chem. 58, 82 - 89 (1993). 